\title{
Survey and Future Directions of Fault-Tolerant Distributed Computing on Board Spacecraft
}

\author{
Muhammad Fayyaz and Tanya Vladimirova* \\ Department of Engineering, University of Leicester LE1 7RH, United Kingdom, \{tv29, mf183\}@1e.ac.uk
}

\begin{abstract}
Current and future space missions demand highly reliable on-board computing systems, which are capable to carry out high-performance data processing. At present no single computing scheme could efficiently tackle high-performance computing as well as reliability. This paper aims to address that gap. In the first part of the paper, a detailed survey of fault-tolerant distributed computing systems for space applications is presented. Fault types and performance parameters for assessment of a fault-tolerant system are introduced. Redundancy schemes for distributed systems are analysed. A review of the state-of-the-art on fault-tolerant distributed systems is presented and limitations of current approaches are discussed. In the second part of the paper, a new fault-tolerant distributed computing platform with wireless links among the computing nodes is proposed. Novel algorithms, enabling important aspects of the architecture, such as time slot priority adaptive fault-tolerant channel access and fault-tolerant distributed computing using task migration are introduced.
\end{abstract}

\section{KEYWORDS:}

Fault-tolerance, Wireless, distributed computing, high-performance, adaptive algorithms, spacecraft.

\section{INTRODUCTION}

In general, a distributed computing system is any computing system that involves multiple processors, remotely located from each other, where each processor plays a particular role in the execution of a computation or control problem. This type of distributed computing is referred to as physically distributed computing. Nowadays physically distributed embedded systems are ubiquitous, having penetrated deeply into our society [1]. Main industry sectors using distributed emebbeded computing are telecommunications, automotive, avionics/aerospace, industrial automation, consumer electronics, health and medical systems. The overall value of the embedded computing market worldwide is about 1600 billion Euro per year [2], with distributed systems accounting for a dominant share of it. The widespread deployment of distributed embedded systems is due to their advantages, namely, high reliability, scalability, suitability for inherently distributed high-performance computing applications [3]. In mission critical applications, such as nuclear power stations or spacecraft systems, for which high reliability is extremely important, the use of distributed embedded computing is a key design consideration. This is mainly because in the event of a single processor failure, the collapse of the whole system could be prevented by distributing the computing load to the redundant processors [4] [5].

An advanced form of distributed computing, which is referred to as cooperative distributed computing, involves collaboration among processors, in which an individual processor solves a part of a larger problem. Cooperative distributed computing is achieved via a distributed computing system that comprises a set of $p$ processors connected via a network. A computing problem is then divided into $t$ tasks, and each processor is assigned a subset of $v$ tasks [6, 7]. Cooperation among processors can make the distributed computing approach more efficient and reliable in comparison with just physically distributed computing units. The ability to cooperatively run a number of tasks in a distributed setting is required by many applications ranging from distributed satellite synthetic aperture radar (SAR) missions $[8,9]$ to autonomous cars [10].

Distributed systems could be more efficient and provide higher performance compared to a single computer

${ }^{*}$ Corresponding author. Tel.: +44 (0)116 252 5372. E-mail address: tv29@le.ac.uk. 
or a set of tightly-coupled computers if the available computing resources are effectively utilized [6] [6,11]. This requires that redundancy is eliminated, wherever possible, however removing redundant nodes can expose processors and networks to faults, leading to degradation of system efficiency and incorrect results. Thus, in a distributed system resources must be dedicated to both solving the computational problem and dealing with system failures.

On-board computing [12] is formed of subsystems, which serve the needs of individual spacecraft subsystems. For fault-tolerance, physical dual redundancy is used, consisting of a primary and a redundant node. This scheme has two main disadvantages. Firstly, all redundant nodes are kept in idle state and are activated only in the case of a failure of its peer node, which leads to inefficient utilization of computing resources. Secondly, the reliability depends on a limited 2:1 redundancy and the failure of both primary and peer node of a critical subsystem may cause the loss of the whole mission. In addition, there are no provisions for resource sharing to support computational performance or fault-tolerance in the context of collaborative distributed systems. Examples of space applications that can benefit from such a distributed model are the following:

- A significant amount of data processing is required for high precision spacecraft control [13]. Current standalone dual redundant computers are unable to process very high data volumes due to physical limitations of current semiconductor technology that restricts the operating frequency of embedded processors [14]. To address these and other requirements of modern spacecraft, the use of distributed computing is essential.

- Synthetic Aperture Radar (SAR), is usually monolithic, i.e. one aircraft or spacecraft is used. A shortcoming of monolithic SAR is its limited resolution. At the same time, distributed SAR, which is based on a cluster of spacecraft, is capable of producing a large synthetic aperture $[15,16]$. Cooperative distributed computing among the spacecraft is necessary to achieve the mission outcomes. A distributed SAR imaging system, based on two satellites, where each satellite can acquire, divide and distribute data chunks to the other satellite for onward transmission to the ground station, is described in [8,9]. Distributed computing in such a system will allow to improve revisit time, image resolution and SAR targeting.

This paper is structured as follows. Section 2 introduces main concepts and redundancy schemes in faulttolerant computing. Section 3 summarises existing fault-tolerant mechanisms of distributed computing systems, focusing on architectural level schemes. Section 4 overviews fault-tolerant distributed systems for space applications, highlighting limitation of existing approaches. Section 5 introduces a novel next-generation spacecraft faulttolerant distributed computing (NGS-FTDC) platform, in which, failures are compensated by migrating tasks to other nodes. Section 6 describes novel algorithms, enabling important aspects of the proposed architecture, such as time slot priority adaptive fault-tolerant channel access and fault-tolerant distributed computing using task migration are also described. Section 7 concludes the paper.

\section{Fault-Tolerant Computing Systems}

In this section, we first introduce faults, fault-tolerance and the performance parameters for evaluation of a faulttolerant computing system and then main redundancy schemes are outlined.

\subsection{Faults and Fault Tolerance}

The terms 'fault', 'error' and 'failure' are extensively used in the context of fault-tolerant systems. A fault is a hardware or software defect that can lead to a system entering into an incorrect state. An error is a part of the system state, which is liable to lead to system failure, while a failure is a state in which the system is restricted from performing its required functions. When designing a fault-tolerant system, a designer makes some assumptions about the types of faults that must be handled. This is denoted as a system fault model. A fault model elaborates all the assumptions of a system failure. A designer will often design a system under the assumption that processors are failed in a fail-stop manner [17].

In general, the following types of faults are often considered [18] [19] [20]:

- Fail-Stop (Fail-Silent) Faults: a processor stops producing outputs when it fails.

- Byzantine (Malicious) Faults: a processor sends erroneous output when it fails. Byzantine fault can be either symmetric or asymmetric. 
- Fail Symmetric: the fault results in the same erroneous value being sent to all other processors.

- Fail Asymmetric: the fault results in different erroneous values being sent to other processors.

Faults may also be classified based on duration:

- Transient Faults: a processor fails and recovers after a short duration.

- Permanent Faults: a processor fails and disappears.

- Intermittent Faults: a processor fails and recovers sporadically.

Faults are also classified as 1) design faults, 2) functional faults and 3) environmental faults [18].

- Design faults are those faults, which are happened due to the immature and bad design practices. These faults can be logic errors or the software errors. These faults can be avoided by hardware testing, by parts screening or by software protections (user / privilege access, user / OS process isolation etc.).

- Functional faults are due to physical failure in component internal structures. These failures are invisible defects, which appear with time or temperature cycling.

- The third types of faults are the environmental faults, which are externally induced error caused by external radiation, electrical noise, or physical damage.

Fault-Tolerance is generally addressed via redundancy, i.e. providing backup resources that can be used in place of a failed resource. Fault-tolerance in a distributed system can be implemented at the architectural level, or at the node level. A redundant node masks failure of a node within a distributed system at the architectural level. At this level, a failed node may exhibits a simple crash or byzantine failure mode. In the optimal case, a node exhibits only a fail-stop failure, i.e. the node is either operational or not. The node implementation must ensure that the failure assumption that has been made at architectural level holds with a high probability at the node level [21].

Selection of suitable performance criteria is vital for the analysis and comparison of fault-tolerant computing systems. We define the following two main criteria: fault coverage and computational integrity for the purpose of performance analysis:

- Fault coverage is measured by qualitative and quantitative parameters. The qualitative parameter specifies the types of faults, which a system can handle while the quantitative parameter represents the conditional probability that a system will recover properly given that a fault of a particular type happens. For each fault type, its quantitative parameter provides a measure of how well the protection mechanism works.

- Computational integrity is measured by three main parameters: (i) Computational accuracy, (ii) Reconfiguration time - a time interval, during which fault isolation and recovery takes place, and (iii) Protection of critical state information. Computational integrity depends on the redundancy scheme, details of which will be covered in 4.3 .

\subsection{Redundancy Schemes}

In a fault-tolerant system, redundancy allows the computer system to operate in the presence of faults or failures. Redundancy can be implemented statically or dynamically. Static redundancy relies on the fault masking approach. In this scheme, a set of multiple processors (triple, quad) are voted to mask a single or double failures. Static redundancy is suitable for applications where maintenance during operation is impractical and is equally applicable to transient and permanent faults [22, 23].

In dynamic redundancy (also called active or standby redundancy), which is an alternative to static redundancy, first, a fault is detected and then a spare unit is substituted in the place of the faulty unit. The rest of this section details main hardware redundancy schemes that have been used in fault-tolerant computing systems.

Simplex Processing with Backup Spares is a dynamic redundancy scheme, which allows a program to run on a single processor, while backup processors are available to take over the task load in a case of a primary failure [24]. Each processor has its own concurrent fault detection mechanism, which enables it to detect faults, e.g. a simple watchdog with Error Detection and Correction (EDAC), a hardware/software Monitor, anomaly detectors. Figure 1 shows a simplex processing redundancy scheme whereby the fault detection is accomplished using 
monitoring of critical signals. The monitoring and reconfiguration module monitors, detects and reconfigures in case of a failure of the primary processor. The safeguard memory stores important data values for the redundant processor.

Standby redundancy can be implemented as warm or cold standby redundancy. In the case of warm standby redundancy, both primary and redundant processors are powered [24]. Normally, only the primary processor executes tasks, while the redundant processor is in its idle state. The downtime of the warm standby redundancy is considerably less because of its backup power state. In cold standby redundancy, the redundant processor is placed in a power down state. In case of failure, the redundant processor is powered up. As the primary and redundant processors execution is not synchronized, a considerable amount of time is required for the redundant processor to reach a fully known state.

There are two main drawbacks of standby redundancy. Firstly, a delay in switching operation loses some of the computation, which gives a lower computational integrity. Secondly, due to single execution, it also has low fault coverage. Nonetheless, it reduces electrical power consumption, and its simple design makes it favourable for long-life missions, such as spacecraft and space rovers.

Duplicated Processing without a Backup Spare is also a dynamic redundancy scheme. It is based on a Dual Modular Redundant (DMR) computer design, in which two processors run the same computation and their outputs are compared. On disagreement, diagnostics are executed to find the failed processor. To identify the faulty processor, it relies on a diagnostic test, which cannot ensure high computational integrity and fault coverage. A fault of an unknown nature can easily be missed, if it is not considered in the diagnostic test routine. However, during the normal operation, it provides a much better fault coverage in comparison to simplex processing.

Figure 2 shows a DMR design, which includes two self-checking (SC) processors. Both self-checking (SC) processors run the same program and have their own EDAC protected data and code memories. Each selfchecking processor has its own fault detection circuit, which can signal an error as soon as it appears. The outputs of the two processors are compared. On disagreement, the processor signals an error is ignored while the other completes its computation. This type of DMR computer has higher computational integrity due to its redundant execution. However, it has a lower fault coverage because of the internal fault detection circuitry that may not detect all errors. In that case, an erroneous output could be delivered to the system.

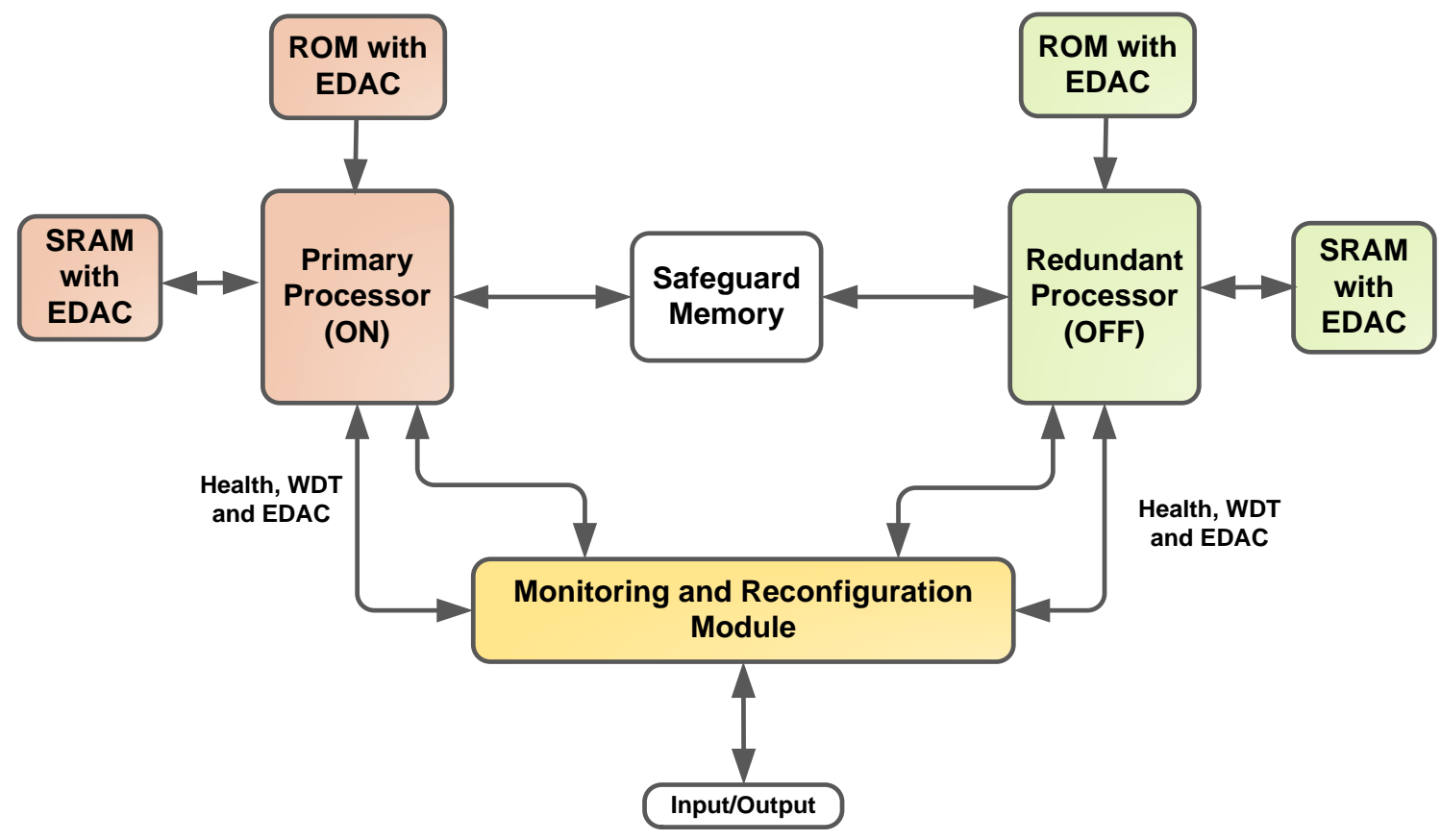

Figure 1: Simplex Processing with a Backup Spare Unit. 


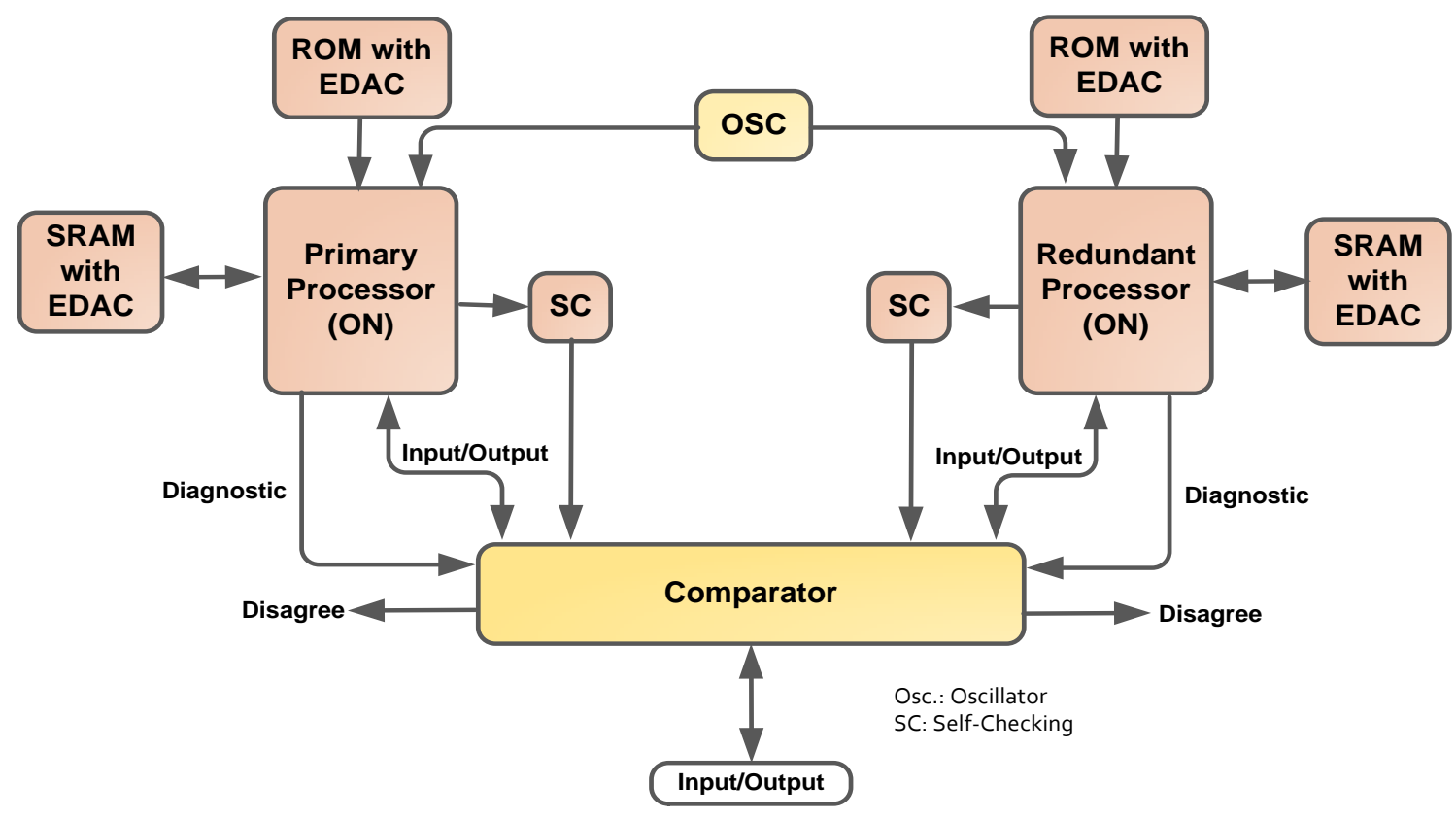

Figure 2: Duplicated Processing without a Backup Spare Unit.

An example of a static redundancy scheme is the popular Triple Modular Redundant (TMR) computer, in which fault-tolerance is provided by using three identical processors. All three processors are clock synchronized, processing the same input information and generating the same output data. The final output, which is delivered to the target system, is derived as a result of a majority voting stage. A voter masks a fault based on majority voting of the processors' outputs [25]. In this scheme, it is assumed that no two processors would fail simultaneously.

TMR computers are conceptually simple, but require a careful implementaion, due to the use of common circuits, such as clock synchronization, voters and interfacing. A failure of the common circuitry in the TMR scheme can be catastrophic, leading to the failure of the whole computer. Therefore, these circuits should be highly reliable. The so-called TMR systems with repair, the usage of which is restricted to small duration missions, are able to integrate the faulty processor back to the system after its successful recovery. However, recovery is a complicated process, which requires all processor register values to be saved before integrating the recovered processor.

The TMR redundancy scheme can only tolerate one failure. If a processor fails in a set of three processors, the fault-tolerance of the TMR computer is no longer effective. To overcome this problem, a hybrid redundancy was proposed in [26, 27], which includes features of both static and dynamic redundancy schemes. This type of redundancy is efficient in terms of fault coverage compared to static and dynamic redundancy schemes, however, additional complexity is added to the computing system design due to the need to manage both types of redundancies.

Three hardware redundancy approaches were described above - dynamic, static and hybrid. In dynamic redundancy, all redundant processors are idle, consuming much less electrical power, which is vital to long-term space applications. In addition, this scheme does not require synchronization of the primary and redundant processors. Furthermore, design diversity between the primary and redundant processors is also possible. The main disadvantages of standby redundancy are the poor fault coverage and computational integrity. On the other hand, TMR computers have a better fault coverage and computational integrity. However, the higher electrical power consumption and other issues such as synchronization and reintegration make them unsuitable for long term missions. The hybrid scheme, which utilizes the features of both, static and dynamic redundancy, includes spares to protect a computer against more than one processor failure. However, designing such a computer is much more complex. Its intricate design increases the design cost and introduces more faults [28-31]. In conclusion, all the above hardware-based redundancy approaches can only protect a system against one processor failure, thus these are only limited to dealing with a single failure. 


\section{Fault-Tolerance Techniques in Distributed Systems}

Redundancy based approaches are limited in terms of reliability as discussed in the previous section. This section presents various fault-tolerance approaches that utilize multiple processors for enhancing the reliability of the overall computing system. The focus of this review is on techniques, particularly designed for reliable distributed embedded systems.

\subsection{Replication Schemes}

Replication involves information sharing to ensure data consistency between the redundant resources. These redundant resources can be hardware or software components. Replication is a well-known solution to faulttolerance management in distributed systems [32]. Replication is initially derived from highly reliable back-end servers where loss of service availability is very critical. Nowadays, it has widespread applications in distributed embedded systems. Two main replication schemes are used: active and passive replication. Variant schemes are semi-active and semi-passive replication, which are derived from active and passive replication, retaining their basic features, respectively.

In the active replication scheme [33], shown in Figure 3, processes are replicated to multiple processors to provide fault-tolerance. The invocating process (client process) addresses the replicas as a process group rather than calling a particular process. After sending a request to all replicas, the invocating process waits for a reply. If the replicas do not behave maliciously, then the invocating process can decide based on the first reply. Otherwise, it waits for at least $K+1$ replicas in a $K$ fault tolerant system. In the first case, the invocating process assumes a fail-silent process failure, while in the second case, it assumes that a Byzantine failure has occurred (behave maliciously when sick). The correct decision in the presence of a Byzantine failure is difficult, and various protocols are used [34-37]. To ensure replica consistency, a totally-ordered multicast mechanism is used. This can be implemented using the Lamport's logical clock that is suitable for small distributed systems. Most of the implementation techniques for active replicas $[38,39]$ assume a partial synchrony of the underlying communication where the messages are communicated within certain time bound limits. In case of a large physical distributed system, a partial synchrony cannot be achievable, and a three-tier architecture is the only solution, as reported in [40-42]. In three-tier architectures, instead of sending a request directly to the replica's processes by the invocating process, an intermediate process is introduced for maintaining the consistency of the replicas.

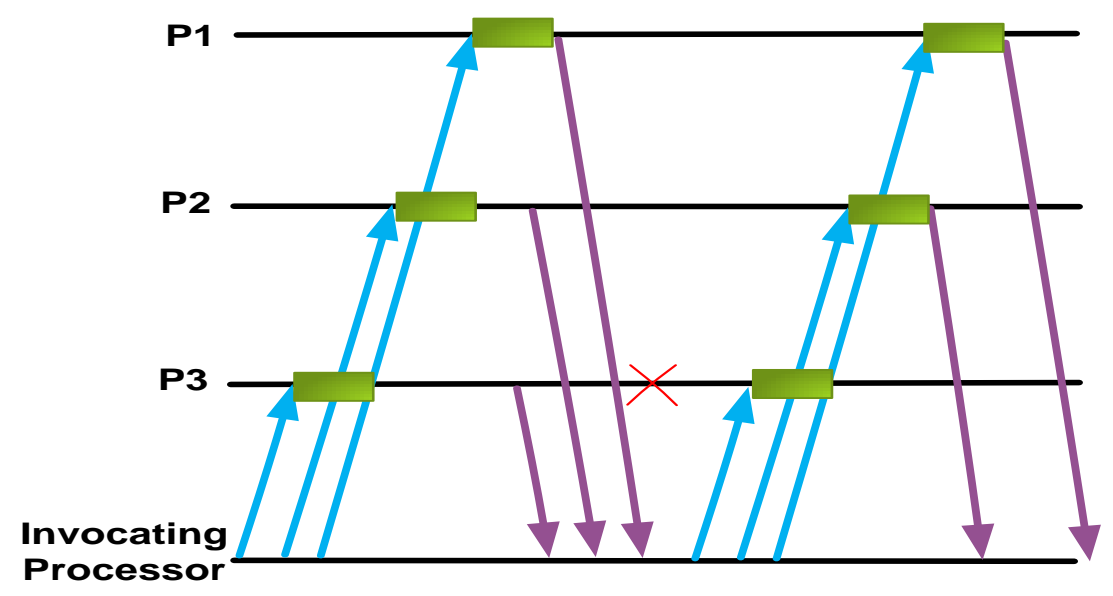

Figure 3: Active Replication.

One important aspect of active replication based reliable system is that they use the method of majority voting, in which a consensus is carried out to ascertain the most reliable outcome amongst each other. However, if one of the nodes fails due to a functional failure or damage, consensus cannot be established, which is a particular limitation in a three-node system. Consensus-based systems also suffer from large inter-node communications overheads, which demand high processing power, consume more energy, and require fast communication net- 
works.

A practical example of an active replication approach is the design of the Maintainable Real-Time System (Mars) [3]. Mars uses active redundancy for fault-tolerance whereby two or more components execute the same tasks. The communication between any two components is also protected against errors by sending messages twice. Components are self-checked and behave silently on the occurrence of a fault. This fail-stop feature restricts components to either sending a correct message or no message. Mars components are arranged in a cluster. The communication between the different components is based upon the time division multiple access (TDMA) scheme.

Another example of active replication is the Delta-4 architecture [43], which consists of multiple computing nodes connected via a local area network (LAN). An individual node can be a uniprocessor, a multiprocessor system or a specialized system comprised of array processors. Software components are replicated to multiple nodes to provide active redundancy against faults or failures. Each node has a network attachment controller (NAC) that provides services related to communication and message self-checking comparison. In addition, the NAC provides multicast and fail-stop node operations.

Active replication is a useful scheme, which is employed in many applications. The main advantages of the active replication scheme are its failure transparency and deterministic timing response to the invocating process. However, this scheme takes a lot of resources, as it requires processing at all nodes to maintain a strong consistency among the replicas.

In the passive replication scheme, only the primary processor processes input messages and provides outputs, as shown in Figure 4. To make the replicas consistent, the internal state of the replicas is regularly updated from the primary processor. So, in the primary-backup scheme, the contacting process communicates only with the primary processor. In a non-blocking primary-backup scheme, the primary processor sends a reply immediately to the contacting process, while in a blocking primary-backup scheme, the primary waits for an acknowledgment from the backup processors before sending a reply to the contacting process. In [44], a trade-off analysis between the blocking and non-blocking scheme is presented. The protocols using a non-blocking primary-backup scheme require a small response time as compared to blocking protocols. Achieving a small response time in point-topoint communications networks is not straightforward, therefore, broadcast communication is preferred in systems implementing non-blocking protocols.

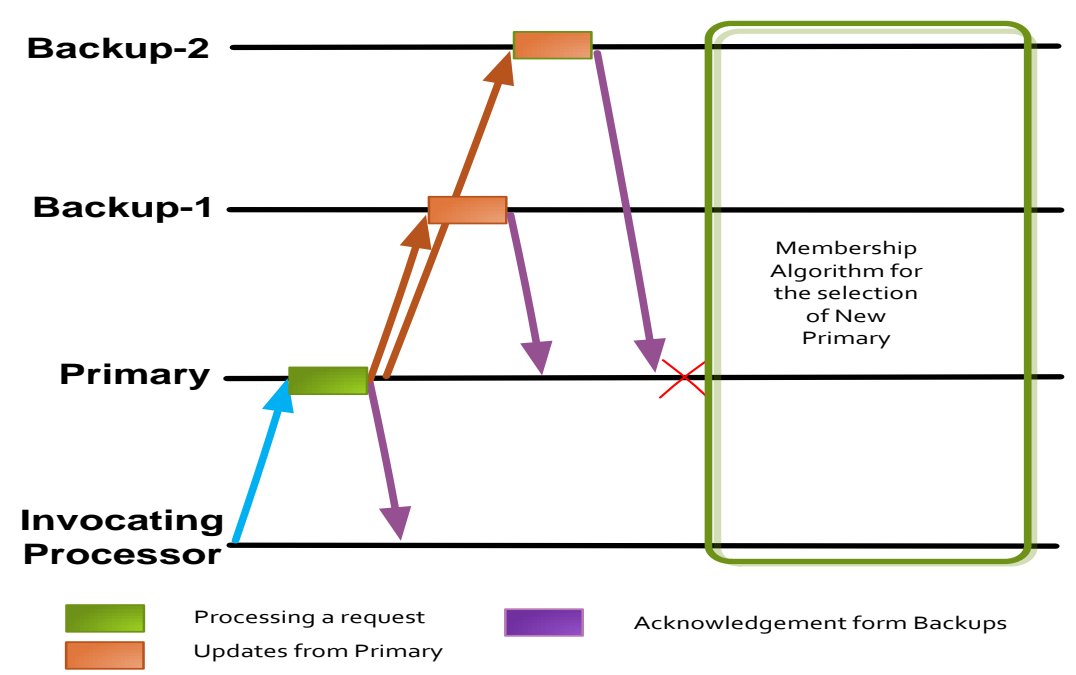

Figure 4: Blocking Primary-Backup Scheme.

A primary-backup scheme for a real-time distributed system is reported in [45], which unlike the active replication scheme, does not require a strong determinism. However, frequent state updates between the primary and the backups are necessary to achieve consistency among the replicas. To achieve a real-time response, a temporal consistency mechanism is employed. Two objects or events are said to be temporally consistent with each other if their corresponding timestamps are within a predefined time interval. In a real-time primary-backup scheme 
the frequency state updates must be compliant with a predefined time bound dependent on the application. This ensures that the backup has sufficient data information to safely replace a failed primary processor in real-time.

Major drawback of passive replication is its slower response to failures in comparison to active replication. Response is particularly slow when the primary replica crashes and a selection of a new primary is initiated, for which a group membership service is required.

In the semi-active replication scheme [46], which is also called "leader-follower", only one replica, i.e. the leader, outputs messages, while the follower replicas perform the same computation autonomously as the leader does, but do not produce an output. However, for a non-deterministic decision, they must follow the instructions of the leader replica, thus relaxing the requirement for determinism.

A new style of replication, called semi-passive replication [47-49], is devised to overcome the slow response of passive replication. In semi-passive replication, a request is sent to all replicas, where only one replica handles the request, after which, it generates a reply message for the client and an update message for the other replicas. Semi-passive replication uses a rotating coordinator [50] for the selection of the new primary rather than a group membership service, thus reducing the amount of time required to selected a new primary. If the primary processor crashes or it is incorrectly suspected of having crashed, then the backup acts as the primary node.

Figure 5 summarises the characteristic features of the replication schemes reviewed above.

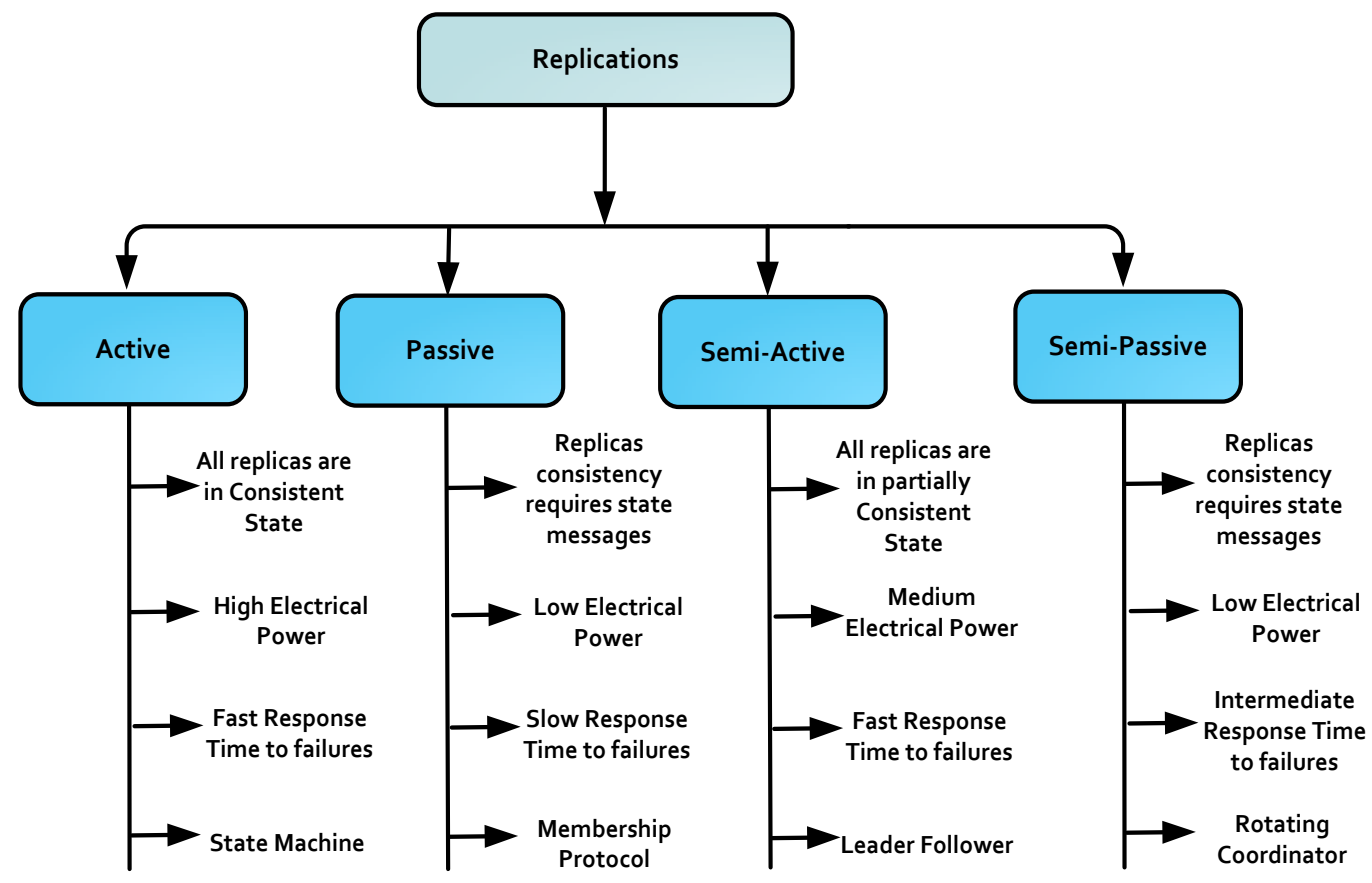

Figure 5: Comparison of Replication Techniques in Distributed Systems

\subsection{Distributed Recovery Block}

In the distributed recovery technique [51,52], two copies of the same program are executed simultaneously on the processors of a node pair, which represents a set of two dual redundant operational nodes. In a node pair, one node is active, called the operational node, while the other node, called the shadow node, is inactive. Under normal conditions, the active operational node executes a primary version of the tasks while the shadow node executes an alternate version of the same tasks. On each node, the correctness of the result is checked by an acceptance test. If the acceptance test is passed, an executive layer routine outputs the results of the primary routine. On failure of the acceptance test inside the primary node, the shadow node is informed either by the primary node executive layer or by the shadow node time-out value (for the case where the primary node fails silently). In that case, the shadow node becomes an active node and sends output results. Similar to the redundancy based approaches, discussed in section 2.2 , this technique has limited reliability and can only protect a computing system against a single processor failure. 


\subsection{Redundant Execution}

Redundant execution (also called time redundancy) is another fault-tolerance technique for distributed embedded systems [53]. Redundant execution can be done at instruction level or task level. In instruction level redundant execution [54], each instruction of the executed program is duplicated and, after each duplicated instruction, results are compared for errors. On the other hand, in task level redundant execution, a software task is executed twice or more in time to avoid temporary faults. Contrary to replications, it does not require additional hardware to run the redundant copy. Instead, it uses extra time to do redundant executions of the same program. As the primary and the redundant executions of a program run on the same hardware, they can only protect the system against transient faults.

A single program executing multiple times reduces the overall computing performance. Additionally, it also consumes more electrical power. However, a form of redundant execution, called multiplexed redundant execution, is able to overcome these problems to some extent, as reported in [55]. The basic scheme is the same as in redundant execution, but a chip multiprocessor (CMP) is used for the execution of leading and trailing threads.

\subsection{Network Surveillance}

Network surveillance is technique that uses a communication network for fault detection and configuration of distributed components [56]. In its simplest form, network surveillance includes a master that periodically makes roll call requests to the other nodes for detection of failures. The master confirms a node failure if it does not receive a reply message. However the central master in this approach constitutes is a single point of failure. A decentralized approach, called periodic reception history broadcast (PRHB), was proposed in [57] to resolve this problem. In PRHB, each node is bound to broadcast periodic reception history, gathered between the last two TDMA cycle. This history corresponds to the health status of the available nodes. The main disadvantage of the scheme is the long latency of the node failure detection, taking up to two TDMA cycles, which increases the response time. Both network surveillance schemes - simple master/slave and PRHB - are used for broadcast networks only. A scheme called supervisory-based network surveillance (SNS) for the point-to-point networks was proposed in [58]. The scheme utilizes two types of nodes - worker nodes and supervisor nodes. Worker nodes pass the health statuses of its neighbour nodes to a supervisor node, which sends status messages to all the other nodes in the network. In this scheme, each node has complete health information of all the other nodes irrespective of the availability of a direct connection. There are two main problems with this scheme: firstly, it requires election of a supervisor in case of thr supervisor node failure, which can take a considerable time; secondly, the messages traverse the network via several links in a store and forward fashion, resulting in additional processing overhead on each node as well as extra sources of faults.

\subsection{Discussion}

In this section a review of the various fault-tolerance approaches that utilize multiple processors for enhancing the reliability of distributed computing systems was presented. The main focus was to analyse the available techniques from the point of view of their suitability for reliable distributed embedded systems.

It is evident that the most widely used fault tolerance technique in distributed systems is replication. The active replication scheme provides a faster response to the invocating process, but it requires a strong replica consistency and consumes more energy due to the execution of multiple replicas. On the other hand, passive replication requires less energy but it does not maintain a full consistency among the replicas. The semi-active replication scheme does not require a strong replica consistency, however, it consumes more energy. Similar to the passive replication scheme, the semi-passive replication scheme requires state messages for replica consistency. In addition, it has a lower response time to the invocating process compared to semi-active replication. To conclude, the replication based schemes are not efficient in terms of resource utilization, as they require excessive computing resources (processors, memory) for the execution and maintenance of the replicas. This makes a replication based distributed system costly and inefficient for resource constrained embedded applications.

In conclusion the reviewed techniques are not well suited for adapting to embedded applications. In addition, the emerging demands for high performance distributed computing require fault-tolerance techniques that utilize the inherent availability of multiple processing units. 


\section{Fault-Tolerant Distributed Systems for SPaCe APPlications}

This section reviews fault-tolerant distributed computing systems aimed at space applications. Existing faulttolerant mechanisms are surveyed to establish the current state of the art. Fault management (FM) schemes as well as fault-tolerant system designs are outlined. Following that modern implementation approaches to faulttolerant computing are summarized and problems of current systems are outlined.

\subsection{Fault Management Schemes}

In general, fault management applies to all parts of a spacecraft, but the focus of this review is fault management at the architectural level, which caters for a failure of a processor, referred to as a computing node in a distributed setting. A computing node's failure can be handled by a manual or an autonomous fault management scheme. In the former FM scheme, a ground command is required for the activation of the redundant node, which leads to a long response time because of the lengthy communication delay and the need of operator intervention. In contrast, the latter FM scheme eliminates this delay by making local decisions on board. There are four main architectural-level schemes for fault management of a spacecraft computing system - half satellite, centralized, decentralized, and hierarchical.

The half satellite FM scheme is the simplest fault management scheme [59], which is usually used for manual on-board configuration. In case of the centralized FM scheme [60], all the functions related to fault detection, isolation and reconfiguration of a system are located on a single computing subsystem called on-board data handling (OBDH). In the decentralized approach [61] [62], the fault monitoring functions are moved to the individual computing nodes. Each node monitors itself and passes the data to the centralized computing node that is the on-board data handling (OBDH) node. Each level has its FM mechanism for failure detection, isolation, and reconfiguration. Instead of a central controller, the hierarchical strategy spreads the fault management functions throughout the spacecraft. This off-loading of the FM functions to multiple units results in a better performance. However, involving multiple levels of fault management makes the overall spacecraft design more complex and vulnerable to failure, if not properly designed. In addition, more efforts will be required on the testing and verification of the FM design.

The Fault-Tolerant Multiprocessor (FTMP) concept is extended to a distributed system in the Advance Information Processing System (AIPS) proposed in [63]. It is divided into multiple groups, whereby each group represents of a triplex, duplex, or simplex redundancy configuration depending upon the criticality of its tasks. These groups are connected via an intercommunication network, which is also triplicated. Each processing group has its local clock for synchronization. Hardware voting is used throughout the system. Hardware voting within a group is easy and managed by the local clock. However, a synchronizer is required for the triplicated data, which are sent among the processing groups.

In $[64,65]$ D. A. Rennels proposed a distributed hierarchical system, which extended the master/slave approach in [66]. The design is comprised of low-level and high-level computers. Each low-level computer has its own task set while the high-level computer is responsible for four main functions - switch on the redundant computer in case of a failure, store ground commands, direct the low-level computers for processing and control of the network communications. One high-level computer is designated to a set of multiple low-level computers. Cold Standby redundancy is used for the low-level computers, while warm standby redundancy is employed for the high-level computers. The high-level computer at one central location handles centralized fault detection, isolation and recovery functions (FDIR) [60]. However, each duplicated low-level computer is dedicated to a particular function and assumed to run only a specific task set. In other words, it cannot reconfigure for other functions, which makes this approach inflexible.

The design of the Maintainable Real-Time System (Mars) started in 1980 [3]. The first Mars prototype targeted for real-time control process applications was developed in 1984 at the University of Berlin. Mars uses active redundancy for fault-tolerance whereby two or more components executes the same tasks. Communication between any two components is also protected against errors by sending messages twice. Components are self-checked and behave silently on the occurrence of a fault. This fail-stop feature restricts components to either sending correct message or no message. Mars components are arranged in a cluster. Within the each cluster, interface component provides extensibility of the cluster. Communication between the different components is based upon the time division multiple access (TDMA) scheme.

The Delta-4 project defines an open, fault-tolerant distributed computing architecture [67]. It is comprised of 
multiple computing nodes connected via a local area network. An individual node can be a processor, a multiprocessor system or a specialized system comprised of an array of processors. Software components are replicated to multiple nodes in order to provide active redundancy against faults or failures. Each node has Network Attachment Controller (NAC) that provides services related to communication and message self-checking comparison. In addition, the NAC provides multicast and fail-stop node operation.

In [68-70] , a task-oriented distributed fault-tolerant computing (FTDC) architecture is proposed, consisting of multiple processing nodes, which execute tasks in a collaborative way, i.e. there are no redundant nodes. Each node in this distributed system, comprises a processing unit and a fault management block, named Adaptive Middleware for Fault-Tolerance (AMFT). Two separate dual redundant networks connected via a bus topology are used for communication between the functional components. This segregation of communication networks does not only reduce the response time in case of a node failure, but also improves the performance of the application tasks. The input/output nodes (I/O), which are attached to the main bus/network, are in dual redundant configurations and are designed to dedicated low level processing related to sensors, actuators, etc.. A distributed fault management strategy is used, which is realized at an architectural level, whereby each node has its own FDIR mechanism, embedded inside the AMFT block. During normal operation, each node is able to monitor itself and send health status information to the other nodes. If no abnormal behaviour is detected, this information confirms that all nodes are in a healthy state. However, in case if any abnormal behaviour is detected by the node itself, a campaign of system reconfiguration is initiated, where all the tasks of the failed node are migrated to other healthy nodes. The fail-over target selection is static and is chosen according to a predefined table, which is stored in each node.

A novel wirelessly connected satellite platform architecture presented in [71] is an extension of the FTDC concept introduced in $[68,69]$. Despite the advantages of wireless networking, current COTS wireless protocols are not designed to meet the required dependability for space applications. The common issues in all protocols, which need to be resolved, are non-deterministic behaviour, non-reliable data delivery, and interference.

A distributed on-board computer architecture, which uses a master/slave FDIR approach, is presented in [72]. The architecture consists of a Master node, an Observer node and Worker nodes, connected to each other via a SpaceWire network. Contrary to the traditional peer redundant scheme, this design supports the concept that a task may run on different targets. Although, the master node is protected by an Observer node, the selection of the next master requires a very robust algorithm. Predetermined decision graphs are used for reconfiguration after a failure. Messages during reconfiguration are broadcast on the same network, which can disturb the normal communication of the healthy nodes. The internal states of the tasks have to be migrated if tasks move to another node due to new mappings or due to a failure of a node. These states are stored on a centralized Storage Interface node, which can be a single point of failure. In addition, due to the centralized storage, a considerable amount of time will be required to transmit the states to the fail-over target node. As the approach uses Master/Worker nodes and all the nodes respond to a single master, there are may be scalability problems, particularly in case of a large number of nodes.

Fault-tolerant distributed computing has been employed in spacecraft in one form or another as detailed above. Three types of approaches can be identified. In first type - AIPS [63] and FTD Computer [64, 65] - multiple redundant processors are used to carry out a set of tasks in existing systems. In the second type - Mars [3], and Delta-4 [67] - hardware redundancy and software replication are employed. In both these approaches reliability is ensured via physical hardware redundancy, which is applied to each processor. Therefore, if one processor fails, its redundant backup takes over, and if that one fails, then there is no provision for transferring its set of tasks to another processor. In these circumstances, the spacecraft has to limit its functionality by operating in a safe mode. The recently proposed third type of distributed computing approaches - Task Oriented Fault-Tolerant Distributed Computing [68, 69, 71] and Master/Slave FDIR design [72], aims to address the aforementioned deficiencies by incorporating distributed coordination for task migration in case of a node failure.

\subsection{Modern Implementation Approaches}

In the following, fault-tolerant distributed systems utilizing reconfigurable, multi-core and multiprocessor approaches are briefly described, highlighting their advantages and disadvantages.

\section{Reconfigurable Fault-Tolerant Computing}

To achieve a reconfigurable computing platform, static random access memory (SRAM) based field programmable gate arrays (FPGAs) are used. Designs implemented using these FPGAs can be modified to tolerate hard- 
ware failures. The reconfigurability feature of SRAM FPGAs is particularly suitable for remote systems, like spacecraft [73].

In [73], a reconfigurable fault-tolerant (RFT) avionics system for a nanosatellite is proposed. A node based on an SRAM FPGA was designed which allows switching between a Simplex and a TMR based redundancy schemes. In [74], another method that includes Simplex, DMR and TMR redundancy schemes is presented. The redundancy scheme is selected based on the severity level of radiation. Each time a pre-set threshold is reached, the corresponding redundant scheme is loaded in the FPGA. During normal operation, it utilizes scrubbing to mitigate the effects of Single Event Upsets (SEUs), where scrubbing is an error correction technique, which is based on rewriting the FPGA configuration to avoid accumulation of errors induced by radiation. FPGAs devices can fail due to two types of failures - physical and functional.

Fault-tolerance due to reconfigurability can only protect the system from functional failures or partial physical failures. In the former case, scrubbing and in-circuit redundancy are employed, while, in the latter case, the design affected by the partial device failure can be relocated to another area of the FPGA programmable fabric. However, the system is vulnerable to a complete device failure, when this approach is used.

\section{Multicore Fault-Tolerant Computing}

Recent trend in on-board computing systems is the use of multicore processors for enhancing the reliability [11]. Multicore processors are inherently redundant in terms of processor cores, $\mathrm{I} / \mathrm{O}$, power supply pins and memory ports that can be used for software or hardware fault-tolerant computing. Software-based fault-tolerance is more suitable for commercial-off-the-shelf (COTS) processors, where hardware modification is impossible. For software-based fault tolerance [75], redundant execution approaches, which exploit the inherent replication of processor cores, are used [76-79]. Redundant execution of a process can be done in both, the time or the spatial domain. A single core is employed to execute multiple copies of the same process in the time domain. This fail-tolerance method is simple, but it is limits to transient faults only. On the other hand, in the spatial domain, multiple cores are used to execute replicated processes. Each replicated process runs on a separate processor core and the outcomes from all cores are compared or voted to produce the final result.

Unfortunately common circuitry of COTS multicore processors, such as clock and control, are not designed to be inherently fault-tolerant. Also, a fault in a single core can stop the whole chip. Furthermore, shared memory among the cores is a potential bottleneck to achieve high performance. Lastly, heat dissipation per small chip, particularly in the presence of vacuum is another issue. To solve these problems, various solutions have been proposed, which include a custom design of multicore processors. For fault containment, one approach is to isolate completely all the cores on the chip. However, each core has its memory controller and input-output connections. Therefore, although fault containment is efficient, hard partitioning of resources such as cache, memory controller and input-output, affects negatively the overall performance. To overcome this problem, a partially partitioned design for a multicore processor is proposed in [77]. This design divides the overall resources into multiple groups, and each group is bound to share its resources. For fault-tolerance, such as in the case of DMR or TMR implementations, each computing core must be from a different group to isolate the primary from the redundant core. A non-shared memory based architecture of a multicore processor is presented in [75], where the latency of shared memory access eliminated. In this architecture, each core has its dedicated memory and sharing of data is accomplished via message passing over a network. In addition, heat dissipation is a problem too, particularly for space applications. Moreover, a single node implemented as a multicore processor is a single point of failure. To address these issues, an on-board fault-tolerant computing system employing distributed multicore processors is introduced in [70], which achieves high reliability and high performance through the inherent availability of multicore processor nodes and a distributed fault detection isolation and recovery (FDIR) scheme.

\section{Cluster-based Fault-Tolerant Computing}

NASA's New Millennium (NMP) ST8 project was aimed at developing a COTS-based dependable multiprocessor systems [80-83]. The architecture consists of dual redundant system controller and multiple data computing nodes, connected to a dual redundant gigabit Ethernet. The whole assembly forms a cluster computer for parallel data processing. Since all type of messages (data, control) are transmitted via a single Ethernet bus, which can potentially delay the cluster's reconfiguration process in case of a failure.

An example of such a system is the Linux-based Beowulf cluster computer for terrestrial applications proposed in [84], which is adopted for a satellite imaging payload application [85]. It comprises 20 StrongARM controllers that are connected by four FPGAs, acting as a parallel processing cluster on board a remote sensing micro-satellite. Heat dissipation is an important issue in embedded cluster architectures for on-board computing 
systems, which requires to be addressed as part of the system design.

The NASA's Remote Exploration and Experimentation (REE) project is also aimed at a fault-tolerant highperformance cluster-based architecture [86, 87]. The main goal is to develop a low cost very high-performance computer system comparable to a supercomputer for deep space missions using COTS components. "The architecture consists of distributed nodes that are interconnected by a fault-resilient system network that supports graceful degradation and has no single point of failure". A multi-level combined hardware and software faulttolerance approach is employed.

\subsection{Limitations of Current Spaceborne Fault-Tolerant Computing}

Legacy spaceborne fault-tolerant computing systems are designed with high reliability as an uncompromised objective, whereby employing redundancy schemes is a common practice. However, using physical redundancy alone can be costly and does not lend itself well to high-performance computing. In addition, recent failures of On-Board Computers, e.g. GOCE in 2013 [88] and Phobos-Grunt in 2012 [89], revealed that centralized and dual redundant computers may fail, resulting in the loss of the complete mission. Further limitations of current fault-tolerant computing systems for space applications are related to computational integrity, adaptability, resource utilization, provision for task migration, isolation of the input-output interfaces, and scalability.

\section{Computational Integrity}

In a fault-tolerant computing system, computation integrity refers to a loss-free computation. Computational integrity can be maintained by periodic storing of computational state data during normal operation and their transfer to the redundant node in case of a failure of the primary node. The review of the state-of-the-art fault tolerance schemes and methods revealed that only a few of them include provisions for storing of computational states on a regular basis. However, in those cases computational integrity was nevertheless compromised due to switching from primary to redundant nodes as well transfer of state data taking considerable time.

Storing of computational states has been achieved through a separate internal module attached to the system bus, or a centralized module connected to the network [90]. However, the former solution adds additional design complexity in terms of isolation between the powered and unpowered nodes, particularly in case of a cold redundant system. On the other hand, the latter provides a complete isolation between the primary and the redundant node, but the state transfer time is considerably high due to the communications involved. Furthermore, both approaches can cause a single point of failure.

\footnotetext{
Adaptability

Important issue is the lack of adaptability in current fault-tolerant systems [91]. In present on-board systems, redundant resources are available only to a particular subsystem, which prevents the sharing of such resources among multiple subsystems. Emerging demands for high performance embedded computing require a faulttolerance technique that utilizes the inherent availability of multiple processing units. In other words, instead of placing idle resources for each subsystem, resources need to be shared for the purpose of high reliability, performance, and computing load balancing.
}

Furthermore, high-performance computing demands of certain space missions vary throughout the mission life, e.g. space probes, space robotics $[92,93]$, etc.. For example, high performance computing is required during the very short landing phase of a planetary Lander for real-time range estimation, as well as terrain visualisation and trajectory calculations. Traditionally to meet these demands additional dedicated processors are used. However, as soon as the landing manoeuvre is over, these computing resources are not utilized for any other purpose. A more efficient solution would be if the system can adapt itself to such scenarios by means of unused computing resources rather than dedicated ones.

Additionally, thermal and radiation design of current high performance space computing systems is rather complex. This could be alleviated, if systems are capable of adapting their operation to compensate for the effects of such environmental factors.

\section{Resource Utilization}

In state-of-the-art redundant spaceborne fault-tolerant computing systems, resources are set aside for faulttolerant operation. For example in the TMR computer, three processors execute the same tasks to achieve high reliability, resulting in a resource utilization efficiency of only $33 \%$. Compared to TMR, the internal redundant centralized and dual redundant standby computers are relatively more efficient, where one processor is dedicated 
to the execution of the tasks, while the other processor is in the idle state, consuming only $50 \%$ of the total available resources.

In traditional physically distributed fault-tolerant computing systems, computing nodes are grouped in pairs of two nodes (primary and redundant) and a node failure is only masked with its peer redundant node. This is why systems which are usually comprised of $n$ nodes require $n$ redundant nodes, whereby the redundant nodes are in the idle state, thus making the system inefficient in terms of resource utilization.

\section{Provision for Task Migration}

Task migration is supported in general purpose distributed computing systems. However, this capability is nonexistent in fault-tolerant distributed embedded systems. The lack of the task migration capability binds the computing tasks to a particular node, thus restricting the computing performance of the system.

\section{Isolation of Input-Output Interfaces}

In fault-tolerant computing systems, both the primary and the redundant computing nodes need to be connected to input-output interfaces transmitting various signals. In case of a failure, a switch-off is essential for isolating a faulty primary node. Although standard methods are employed for input-output isolation of the primary and redundant nodes [94, 95], the powered-off faulty node can accidently be back-powered from the common logic signals, thus causing unexpected behaviour in the operating node.

\section{Scalability}

Current on-board fault-tolerant computing systems are physically bound to a certain input / output sizing. Considerable hardware modifications are required in order to increase their system level reliability and performance. Therefore, these systems do not lend themselves well to scalable data-intensive computing applications.

\section{NeXt Generation SpaCecraft Fault-Tolerant Distributed Computing Platform}

The need for more advanced space missions is growing. Spacecraft systems are constantly evolving with the ongoing developments, advances, and innovations in various fields of modern technology. In future, the trend towards spacecraft sophistication will continue to grow. In order to meet adequately the heightened needs of future missions it is uttermost import to devise on-board computing systems featuring high reliability, adaptability, and scalability. The initial concept of a fault-tolerant distributed computing on-board architecture using task migration for fault-tolerance $[68,69]$ was introduced in section 4.1. In this section a next-generation spacecraft faulttolerant distributed computing (NGS-FTDC) platform is proposed, which is an extension to the FTDC concept.

\subsection{Proposed Computing Model}

The proposed design of a NGS-FTDC platform for future space applications is shown in Figure 6. It comprises distributed nodes and a network. There are two types of nodes: computing nodes and Input-Output (IO) nodes. A computing node is a self-contained entity which has its own fault detection, isolation and reconfiguration block called Adaptive Middleware for Fault-Tolerant block $[68,69]$ while the IO node is responsible for sensor data acquisition and commanding. The reconfiguration in case of a failure is supported by a novel algorithm (section 6.2) for migrating computing tasks to other nodes. The computing node is implemented as a multiprocessor system-on-chip (MPSoC) that includes multiple high-performance processors running application tasks and a specialized highly reliable processor for the implementation of AMFT. Providing only the AMFT processor as a highly reliable component, ensures that the most critical functionality of the node is protected from SEUs and keeps the cost of the node down.

This paper broadens the wireless FTDC model in [70]. Wireless communication provides advantages over wired communication reducing substantially the satellite harness mass and bulk as well as decreasing vulnerability of communications to space debris impact via elimination of connecting wires as possible points of failure. The size of the MPSoC-based distributed computing nodes is very small and due to the wireless links they could be placed anywhere in the spacecraft. For illustration purposes, two types of networks, WiFi and ZigBee, are employed in Figure 6. WiFi is utilised for the main communication of the On-Board Data Handling (OBDH), Attitude \& Orbit Control System (AOCS), and the communication subsystems. The ZigBee protocol is used for two purposes: firstly, for the data acquisition of the health sensors and secondly, for the communication among the 
AMFT blocks [70]. A novel algorithm (section 6.1) facilitates a time slot priority adaptive fault-tolerant channel access, addressing the non-deterministic behaviour of wireless protocols.

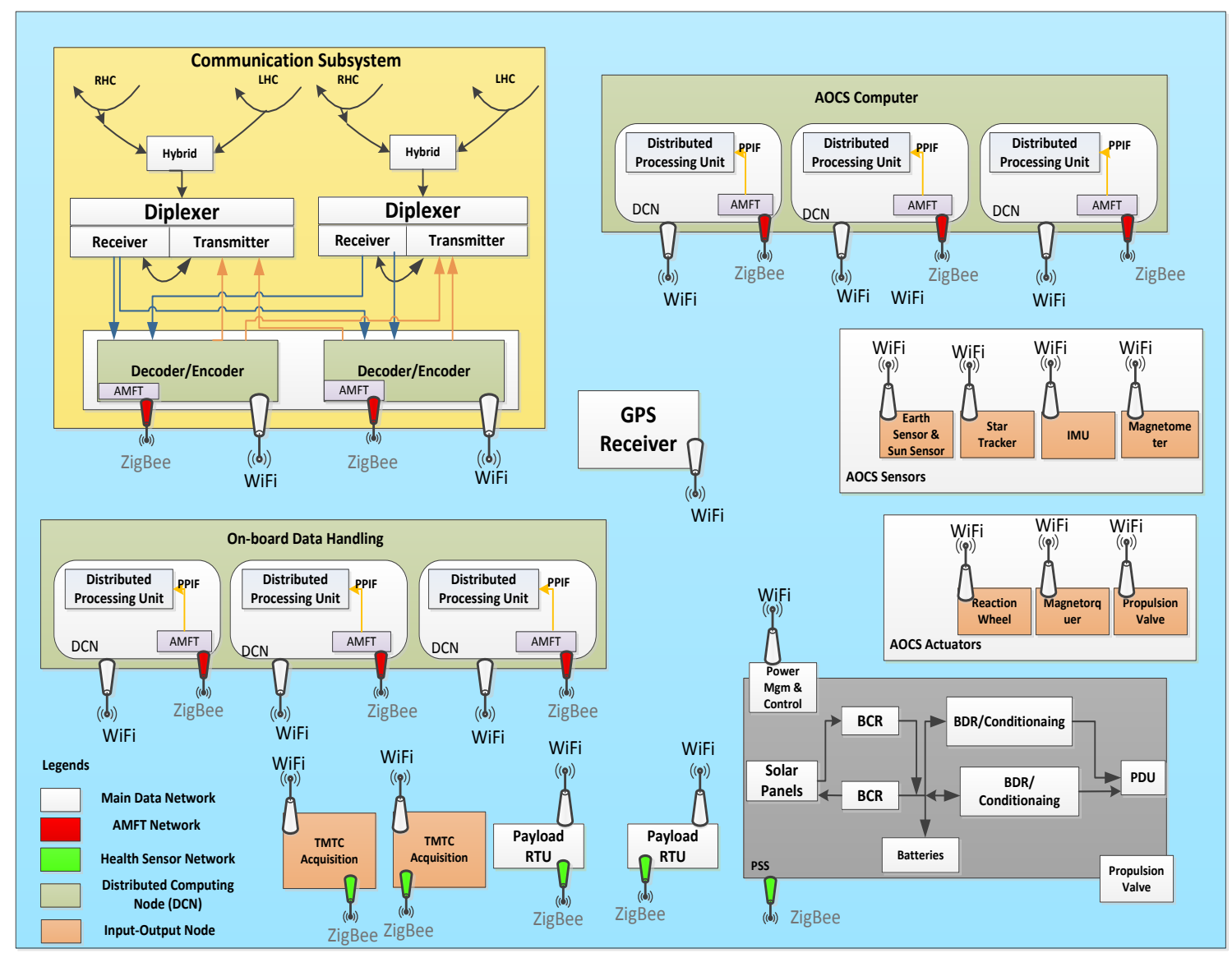

Figure 6: Next Generation Spacecraft Fault-Tolerant Distributed Computing Platform

\subsection{Addressing Needs of Future Spacecraft Missions}

The proposed NGS-FTDC platform not only addresses the limitations discussed in section 4.3, but also provides additional benefits to future spacecraft, as detailed below.

- Computational Integrity: Contrary to traditional approaches, a node failure in the proposed computing platform is compensated by migration of computing tasks. Task migration requires a much shorter reconfiguration time in comparison to switching between nodes. The small reconfiguration time indirectly increases the computational integrity of the proposed computing platform.

- Adaptability: Adaptability defines the capability of a computing system to adapt itself in response to occurring changes. A spaceborne computing platform should also be able to cope with high workloads and faults. Under a fault condition, the proposed computing platform is capable of adapting itself to a healthy configuration, while in case of a high computing load, it is able to optimise the tasks execution in order to avoid a complete halt in the system.

- Provision of Task Migration: A cheaper and better approach for future space missions are distributed computing systems, comprised of multiple computing nodes, which do not employ hardware redundancy. For fault-tolerance, computing resources are intelligently used and the recovery after a node failure is provided by migrating tasks to other nodes. In this approach, rather than reserving redundant resources for each computing node, the computing resources are reconfigured making use of the present multiple nodes. Consequently, the proposed approach is resource efficient in terms of overall utilization of redundant resources. In 
addition, it is inherently fault-tolerant due to mapping of an application to multiple processors, which allows a degraded service even in the case of failures. Furthermore, degraded service is restored to full functional service by migrating tasks to other computing nodes. Tasks are migrated to more than one node, thus acheiving high reliability compared to traditional redundancy.

- Scalability: In order to accommodate evolutionary technological advances, space distributed computing systems will have to be scalable. In general, scalability of distributed systems refers to the addition of computing nodes in an existing system. However, scalability applies to all components, e.g. the computational problems, fault-tolerance mechanisms and communication networks.

The proposed NGS-FTDC platform provides a scalable and extendable computing without the need of redesigning and remapping of the entire functionality. It is scalable in terms of its fault-tolerance capability. Additional computing nodes can be added or dropped for the sake of achieving fault-tolerance on the fly without compromising the overall reliability of the distributed computing system. Furthermore the wireless network, which provides the communication core of the proposed platform, is extendable and scalable.

- Resource Sharing: It is envisioned that future spacecraft computing systems will be required to support several applications running simultaneously which will necessitate sharing of computing resources and processed data among them. The proposed computing platform lends itself well to this need, as it supports sharing of computing resources among nodes for the purpose of achieving both high performance and faulttolerance. Therefore, a high-performance computing node would be able to share its computing resources, whenever a demand is raised from a low-performance computing node. In addition, duplicated data processing at multiple nodes is avoided by sharing of the processed data.

Several technologies for resource sharing have been implemented in terrestrial distributed computing systems, for example, remote procedure call (RPC), Common Object Request Broker Architecture (CORBA), Distributed Component Object Model (DCOM), Remote Method Invocation (RMI) and XML-RPC. Incorporation of such technologies in the proposed computing platform will be highly beneficial to space highperformance computing applications, particularly in cases where the computation to communication ratio will be very high.

- Wireless Network: Introduction of wireless connectivity in satellites makes on-board subsystem designs more flexible. It allows a subsystem to join or leave the network without the need of changing the interconnections among the different subsystems. No special connectors are needed for additional diagnostics and data links are inherently immune to wear and tear. In addition, wireless connectivity will speed up and make easier system integration, testing and operational diagnostics in future satellite systems of ever increasing complexity comprising many integrated subsystems. Furthermore, design reusability, upgrade, and relocation of subsystems will become simpler, since there is no need to re-route data cables.

- Isolation of Input-Output Interfaces: In the proposed computing platform transfer of data among the nodes is carried out over a wireless network, and all node inputs and outputs are inherently isolated due to the nature of wireless communication.

\subsection{Discussion}

The development of the above NGS-FTDC platform, which is based on wireless communication, is quite challenging [96] [97], despite recent considerable advancements in wireless technologies and processor chips. Below are discussed some of the challenges faced by designers.

In case of a failure of a node in the FTDC system, fault detection, isolation and reconfiguration activities will be carried out. Each node should be able to detect and isolate itself from the rest of the distributed system. This step will require a highly reliable local fault detection process which can only be achieved if detection algorithms are executed on a separate reliable hardware. Once a fault is detected, a reconfiguration process will be started, whereby tasks are migrated to other nodes. If the tasks are of a critical nature, they have to be resumed from their pre-emption points. Furthermore, each task requires a copy of its memory space. These necessitate that sufficient storage is available to keep the pre-emption points and a local copy of the memory space.

Space environmental hazards, such as high levels of radiation, extreme temperatures, electromagnetic interactions of space weather, etc., could significantly affect the channel quality, posing significant challenges to the on-board wireless communication. Degradation due to the time-varying nature of flat fading and frequency se- 
lective fading could also temporary disrupt the communication signal. Due to these effects, the wireless communication channel can exhibit unreliable data transmission behaviour. Preserving data integrity will require a repeated multiple transmission or error correcting codes.

Electromagnetic Interference (EMI) is considered a major issue in utilization of wireless technologies for space applications [98]. Interference can be due to intra-interference, caused by the wireless network, or it can be due to inter-interference caused by other on-board subsystems. Intra-interference can also be due to reflected interference or adjacent channel interference. Reflected interference is provoked by the closed metallic structure of the spacecraft, whereby a signal is reflected several times. This can cause damage to the wireless interface if not controlled properly. While the adjacent channel interference is not severe and can easily be controlled by existing methods (sharp filtering, and orthogonal codes). Each subsystem on the spacecraft emits radiation from $0 \mathrm{~Hz}$ to several $\mathrm{GHz}$. However, the magnitude of most of these interferences is very small except for the radio frequency (RF) equipment, which is particularly designed to radiate RF output power at a certain frequency. So, to establish a successful intra-satellite wireless communication, a proper frequency selection between the existing RF and intra-satellite wireless equipment is essential.

For the purpose of resource sharing, software components need to be developed, which can easily be mapped to the low memory space of embedded computing systems [99]. In addition, these should be flexible to allow additional functionality.

\section{Enabling The NGS-FTDC Platform}

This section presents two new algorithms, which aim to contribute to the realization of important aspects of the NGS-FTDC platform, proposed in section 5. The first algorithm provides a deterministic wireless channel access and ensures reliable data delivery among the computing nodes. Both properties are essential for on-board spacecraft computing via wireless communication. The second algorithm specifies a node level fault-tolerance and reconfiguration mechanism, following a node failure or a recovery. Table 2 describes the symbols used in both algorithms:

TABLE 1: TERMS USED IN THE DESCRIPTION OF THE PROPOSED ALGORITHMS

\begin{tabular}{|c|c|c|c|c|c|}
\hline $\begin{array}{l}\text { Sym- } \\
\text { bol }\end{array}$ & Description & Symbol & Description & Symbol & Description \\
\hline$n$ & A node & $n_{r}$ & Receiver node & $n_{s}$ & Sender node \\
\hline ts & $\begin{array}{l}\text { Communication } \\
\text { Time Slot }\end{array}$ & $p_{v}$ & $\begin{array}{l}\text { Node Channel } \\
\text { Access Priority }\end{array}$ & $\forall$ & For all \\
\hline Dpkt $_{\text {set }}$ & Data Packet Set & Dpkt $_{p}$ & $\begin{array}{l}\text { Data Packet Pri- } \\
\text { mary }\end{array}$ & Dpkt $_{r 1}$ & $\begin{array}{l}\text { Data Packet Re- } \\
\text { dundant-1 }\end{array}$ \\
\hline Dpkt $_{r 2}$ & $\begin{array}{l}\text { Data Packet Re- } \\
\text { dundant-2 }\end{array}$ & Ack $_{\text {set }}$ & $\begin{array}{l}\text { Acknowledge- } \\
\text { ment Packet Set }\end{array}$ & Ack $_{p}$ & $\begin{array}{l}\text { Acknowledge- } \\
\text { ment primary }\end{array}$ \\
\hline $\operatorname{Ack}_{r 1}$ & $\begin{array}{l}\text { Acknowledge- } \\
\text { ment redundant- } \\
1\end{array}$ & $A c k_{r 2}$ & $\begin{array}{l}\text { Acknowledge- } \\
\text { ment redundant-2 }\end{array}$ & $p_{\text {highest }}$ & Highest Priority \\
\hline$p_{v}^{\text {backoff }}$ & \multicolumn{3}{|c|}{$\begin{array}{l}\text { Back off Priority, a priority value which is subtracted } \\
\text { from the actual value, if there is a collision. }\end{array}$} & $N$ & $\begin{array}{l}\text { Total Number of } \\
\text { nodes }\end{array}$ \\
\hline$n_{\text {id }}$ & Node ID & $t_{s e t 1}, t_{s e t 2}, t_{s e t 3 \ldots}$ & Task set & $\begin{array}{l}t_{\text {set } 1}, t_{\text {set2 } 2 . . T L_{o}} \\
p\end{array}$ & List of Task sets \\
\hline$\Delta_{S D 1}$ & Task-1 State Data & $H B$ & \multicolumn{3}{|l|}{ Heartbeat Message } \\
\hline$R R$ & \multicolumn{2}{|c|}{ Request for registration } & $\in$ & $\begin{array}{l}\text { Belongs } \\
\text { to }\end{array}$ & $\begin{array}{l}\text { A faulty } \\
\text { node }\end{array}$ \\
\hline
\end{tabular}

\subsection{Time-Slot Priority Adaptive Fault-Tolerant Channel Access Algorithm}

The wireless channel is partially synchronous and has poor data reliability. Packets get lost, and timely delivery cannot be guaranteed in distributed computing systems. In order to cope with these problems, a Time-Slot Priori- 
ty Adaptive Fault-Tolerant Channel Access (TSPA-FTCA) algorithm is proposed. The following are the characteristic features of the algorithm:

- Adaptive Node Priority: Each node $n$ has its own priority value, which provides access to the communication channel. The priority value is changed adaptively according to the use of the channel. The priority value varies from 0 to 1024, with ' 0 ' being the lowest priority while '1024' - the highest. On each successful access of the communication channel, a node $n$ consumes 64-points of its priority, $p_{v}$, which means that the node's priority is decreased by ' 64 '. However, a node $n$ gets 8 -points, whenever any other node has a successful access to the channel or the slot is not used by any other node.

- Fixed Slots for Communication: The communication channel is divided into time slots $t s$ of a fixed length. Each node can only communicate during a time slot. During the transmission of a node $n$ within a particular time slot, no other node can have access to the channel. A prioritized timed channel access ensures deterministic access to the channel, as compared to the current Carrier Sense Multiple Access/Collision Avoidance (CSMA/CA) wireless communication protocol. Deterministic access is essential for critical applications.

- Packet Loss and Data Reliability: In wireless communication, due to the varied nature of the channel, data reliability cannot be assured. In the proposed algorithm, information redundancy is used to tolerate packet loss or errors in the wireless communication channel. Loss or errors in the wireless communication channel cannot be avoided. Therefore, a scheme, which involves triplication of the packets, is proposed. In this scheme, a node sends its data packet set $D p k t_{\text {set }}$, comprised of a primary packet $D p k t_{p}$ and two redundant packets $\left(D p k t_{r 1}, D p k t_{r 2}\right)$. This triplication of packets ensures the data reliability over the wireless channel.

- Local View of Priority Table: Each node $n$ maintains a local view table, which stores the priority values for all nodes. All the nodes except the sender node $n_{s}$ update their local view table on receipt of the local view message.

\subsubsection{TSPA-FTCA Algorithm Description}

A distributed computing system is assumed, which comprises $N$ nodes, where each node executes the TSPAFTCA algorithm for deterministic access to the communication channel. The functions and logic flow of the TSPA-FTCA algorithm are represented by the pseudo code in Listing 1, which is detailed below:

Lines 1-6: These implement the initialization of the variables for a node $n$, initializing mainly the priority table, data packet and acknowledgement packet. At the time of initialization, all nodes' priorities are set to the highest priority value of 1024 .

Lines 7-22: Before a node $n$ transmits data on the communication channel, a highest priority node is searched in the local view of the priority table. A local view is a priority table, which shows the priorities of all $N$ nodes of the distributed system. This information is acquired from the periodic transmission of local view messages. If all the tables are fully synchronized, a highest priority $p_{\text {highest }}$ node can be easily found. If multiple nodes have the same priority, then the decision for access to the transmission slot is resolved based on the node ID $n_{i d}$ (lines 2122). The node with the lowest ID will win. The chances of a collision are very low in the proposed algorithm, however, if a collision happens, it is resolved by finding a new priority value (line 14-16). A new priority value is obtained by subtracting the existing priority value $p_{v}$ from the back off priority value $p_{v}{ }^{\text {backoff. }}$. This value is only updated for the nodes, which are involved in the collision, and only the local view tables of these nodes are updated. In cases of no collision, a sender node $n_{s}$ sends a packet and receives an acknowledgement from the recipient node $n_{r}$.

Lines 23-31: All the other $N-1$ nodes receive a data packet. For each received triplicated data packet set, $D p k t_{\text {set }}$ (consisting of one primary $D p k t_{p}$, one redundant data packet-1, $D p k t_{r 1}$, and one redundant data packet-2, $D p k t_{r 2}$ ) a bitwise TMR voter is used to mask the errors at the first place, and then the resultant packet is further checked for errors using a Cyclic Redundancy Check (CRC). If there is an error, the packet is discarded, otherwise, it is stored for later forwarding to the application layer. TMR voting cannot be possible, if $2 / 3$ or $1 / 3$ of the component packets are lost during the transmission. For these two cases, the remaining received packet/packets, are only checked for CRC errors and if there is no CRC error the packet is retained, otherwise it is discarded.

Lines 32-33: Each node $n$ periodically sends a local view of its priority table in a local view message. All the other $N-1$ nodes receive this message and update their priorities with the currently received values. This helps to synchronize all the local view tables and ensures consistent priority values inside all the tables. The more these 
tables are synchronized (all having the same local view), the few collisions will occur on the communication channels. Based on the priority value, a node $n$ knows about the next communicating node.

Lines 34-35: All other $N-1$ nodes except the sender node $n_{s}$ increase their priority $p_{v}$ by adding ' 8 ' to the current priority value.

\section{Listing 1. TIME-Slot PRIORITY ADAPTIVE FAUlt-ToleRANT ChANNEl ACCESS (TSPA-FTCA)}

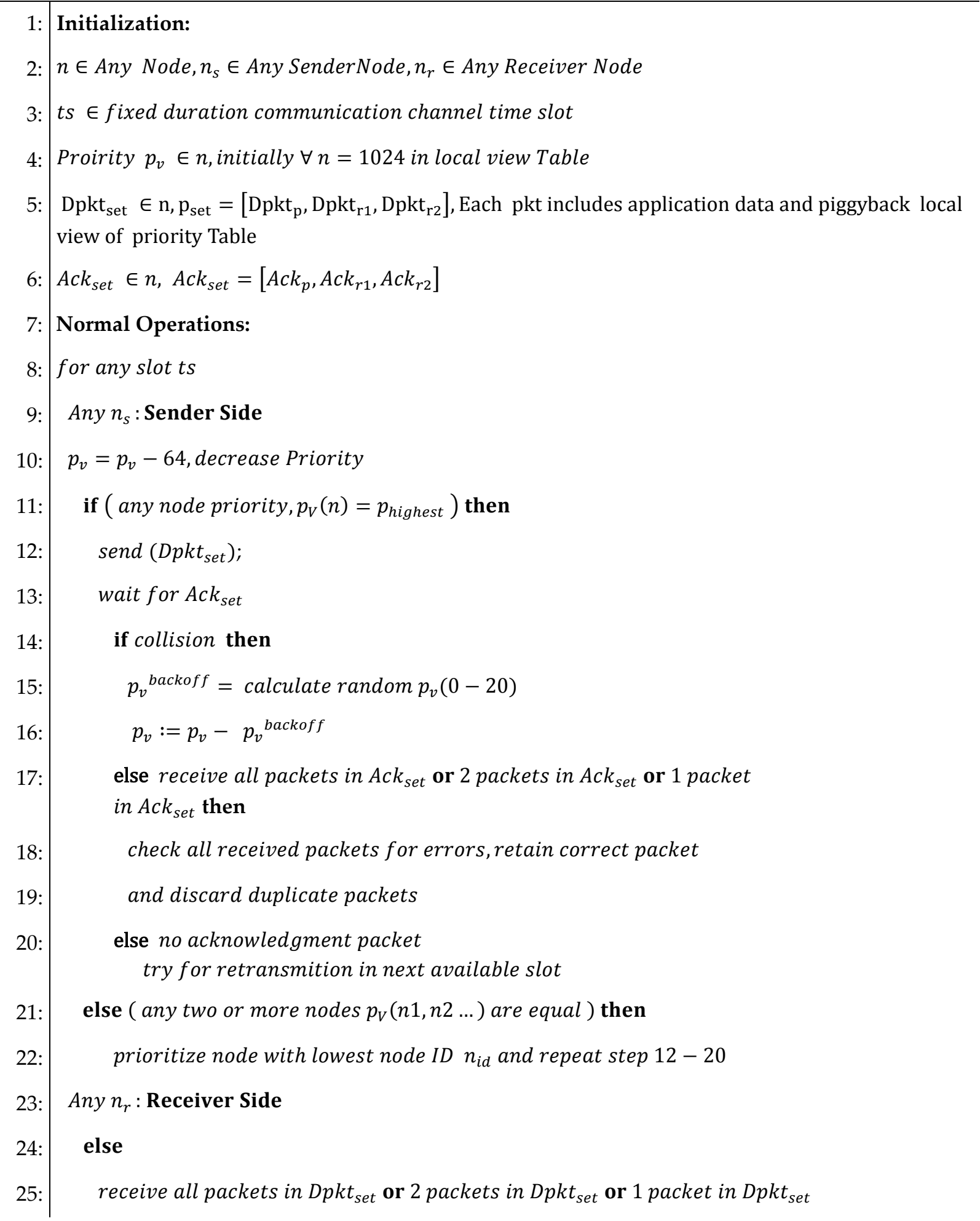




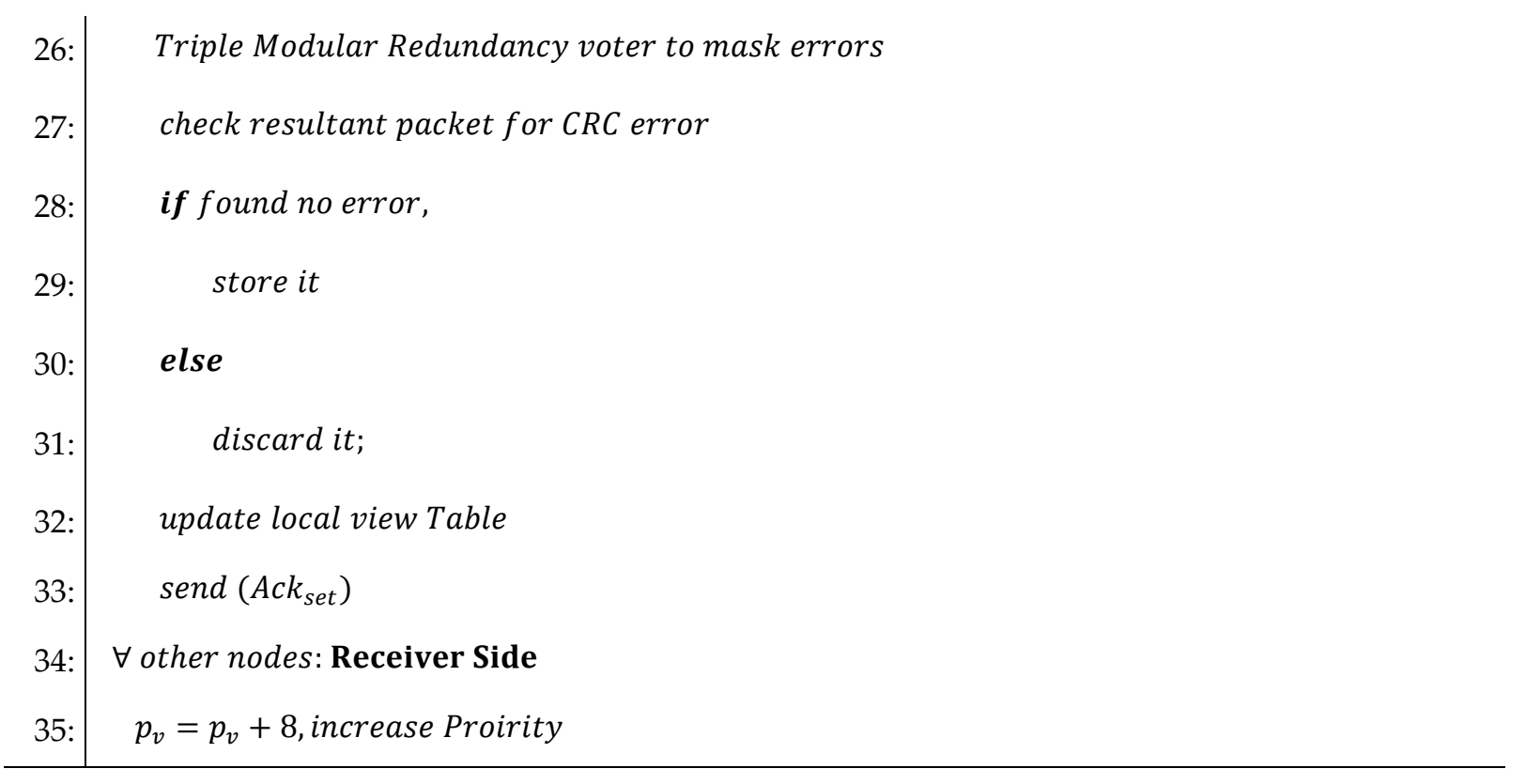

\subsubsection{Operational Scenarios}

In this section, three operational scenarios are tested to prove the correctness of the TSPA-FTCA algorithm. The scenarios include a single node, two nodes and three nodes. For each scenario, results with the same and different initial node priority values are obtained to demonstrate that the algorithm converges for both cases. The algorithm is executed for 100 time slots with time slot duration of $1 \mathrm{~ms}$ in both cases (same priorities and different initial priorities).

\subsubsection{Single-Node Operational Scenario}

In the single node scenario, only a single node is active. Figure 7 shows the obtained results, when the algorithm is executed for a single node configuration. The upper part of Figure 11 shows the results, when the algorithm is executed for an initial priority of 1024, while the lower shows the results when it is executed for a priority $p_{v}$ of 400. The obtained results show that the algorithm is independent of the initially selected priority. In both cases, on each successful slot access, the priority $p_{v}$ is decreased by ' 64 ' until it reaches the lower limit of ' 0 '. At this stage, further transmission of packets is ceased until its priority $p_{v}$ dynamically changes to a value higher than or equal to 64 , which is the required minimum priority for transmission of a packet on the communication channel. In both cases, as soon as the priority $p_{v}$ of a node is dynamically changed to 64 , the node accesses the channel and its priority again decreases to ' 0 '.

\subsubsection{Two-Node Operational Scenario}

In case of a two-node operational scenario, first both nodes are configured with the same priority value $p_{v}$ of ' 1024 '. The same priority $p_{v}$ is chosen to show the collision avoidance mechanism, when two nodes of the same priority attempt to access the channel simultaneously. A collision can only happen, when the local view priority tables of the nodes are not synchronized. In that case, the nodes, which cause the collision, change their priorities using a back-off mechanism (lines 15-17 in Listing 1). Table 2 shows the priority tables for node- 1 and node-2, which have different local views. According to the node-1 local view, node-1 has high priority, while as per the node-2 local view, node-2 has high priority. Due to the different local views of the priority tables, a collision occurs, which is handled by the back-off mechanism of the algorithm. During the normal operation, due to the periodic transmission of local view messages for priorities tables, all the nodes develop consensus and have the same priority values in the local view tables. In a scenario, when two or more than two nodes have the same priority value, then the node which has the lowest node ID $n_{i d}$ will be the first to transmit on the communication link.

As shown in the upper part of Figure 8, although both nodes have started with an equal priority of 1024, due to the collision, the priorities are set dynamically. Both nodes can access the communication channel based on their 
priority, however access to the channel is denied when the priority value $p_{v}$ is equal to zero. Following that, priority increases steadily up to 64 to allow a channel access again. In the lower part of Figure 8, different initial priorities are used, showing that node- 2 cannot access the channel until its priority value is greater than that of node-1.
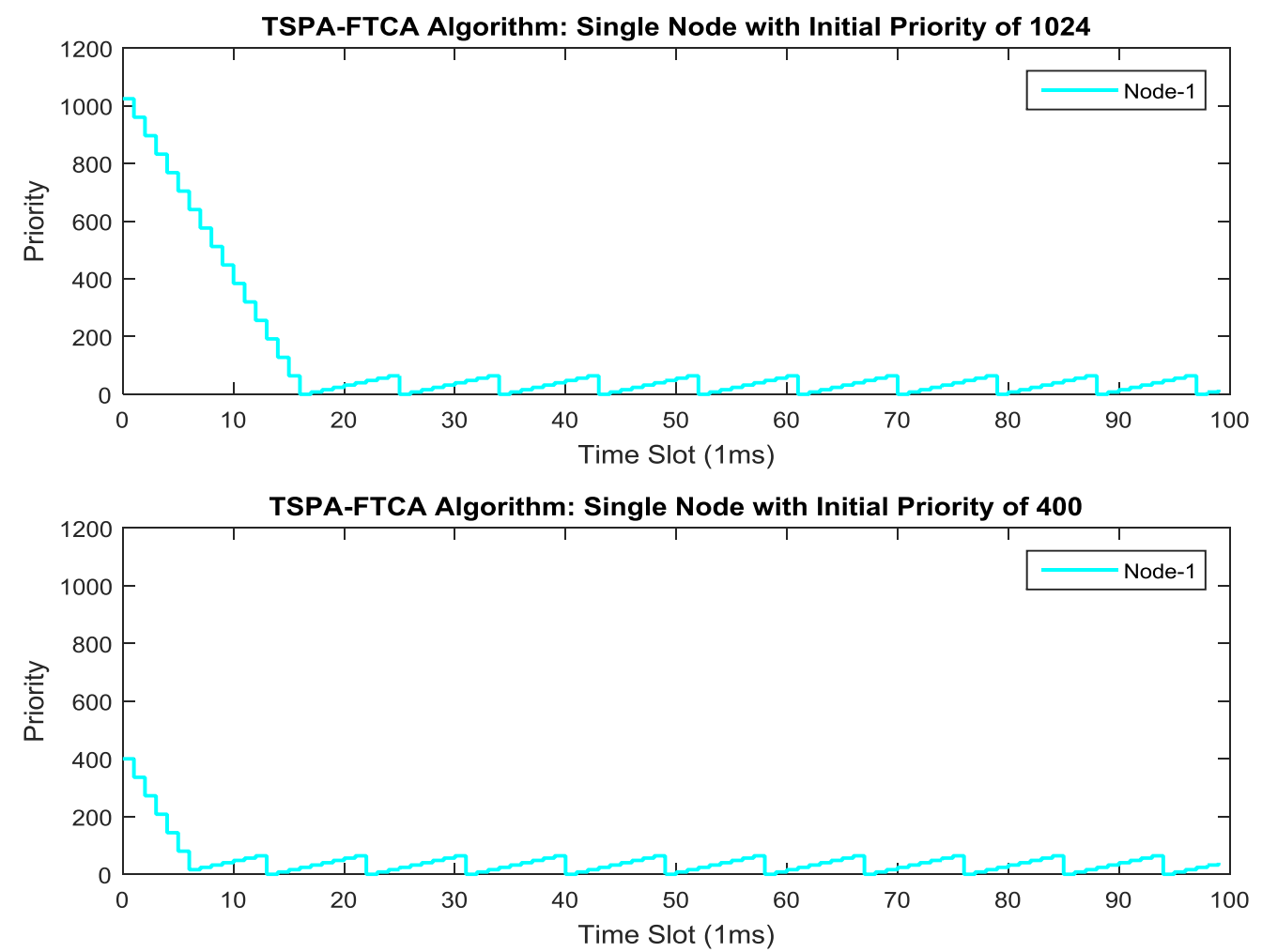

Figure 7: TSPA-FTCA Algorithm in a Single Node Configuration.

TABLE 2: LOCAL VIEW OF PRIORITY TABLES

\begin{tabular}{|c|c|c|c|c|c|}
\hline \multicolumn{6}{|c|}{ Before Collision } \\
\hline \multicolumn{3}{|c|}{ Local View of Priority Table [Node-1] } & \multicolumn{3}{|c|}{ Local View of Priority Table [Node-2] } \\
\hline Node ID & Priority & & Node ID & Priority & \\
\hline 1 & 1024 & High Priority Node & 1 & 1010 & \\
\hline 2 & 1000 & & 2 & 1024 & High Priority Node \\
\hline \multicolumn{6}{|c|}{ After Collision } \\
\hline \multicolumn{3}{|c|}{ Local View of Priority Table [Node-1] } & \multicolumn{3}{|c|}{ Local View of Priority Table [Node-2] } \\
\hline Node ID & Priority & & Node ID & Priority & \\
\hline 1 & 1018 & High Priority Node & 1 & 1010 & High Priority Node \\
\hline 2 & 1000 & & 2 & 1008 & \\
\hline
\end{tabular}

\subsubsection{Three-Node Operational Scenario}

The three-node operational scenario in Figure 9 shows that the performance of the algorithm is not affected by 
the increase of the number of the nodes. Based on the priority among the nodes, the node with high priority $p_{\text {highest }}$ is the first that can access the channel. Similarly to the two-node scenario, same and different initial priorities values were considered. For both cases, the channel access is allocated fairly among the nodes.
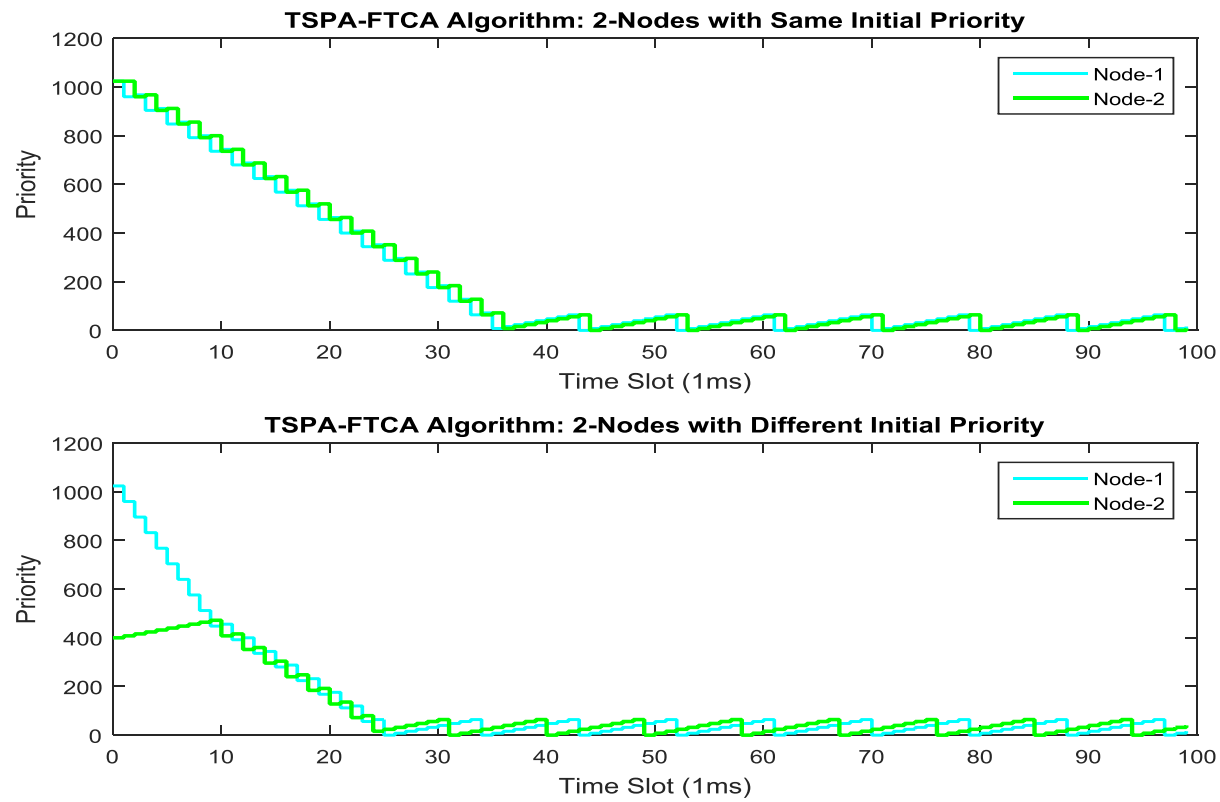

Figure 8: TSPA-FTCA Algorithm in a Two-Node Configuration.
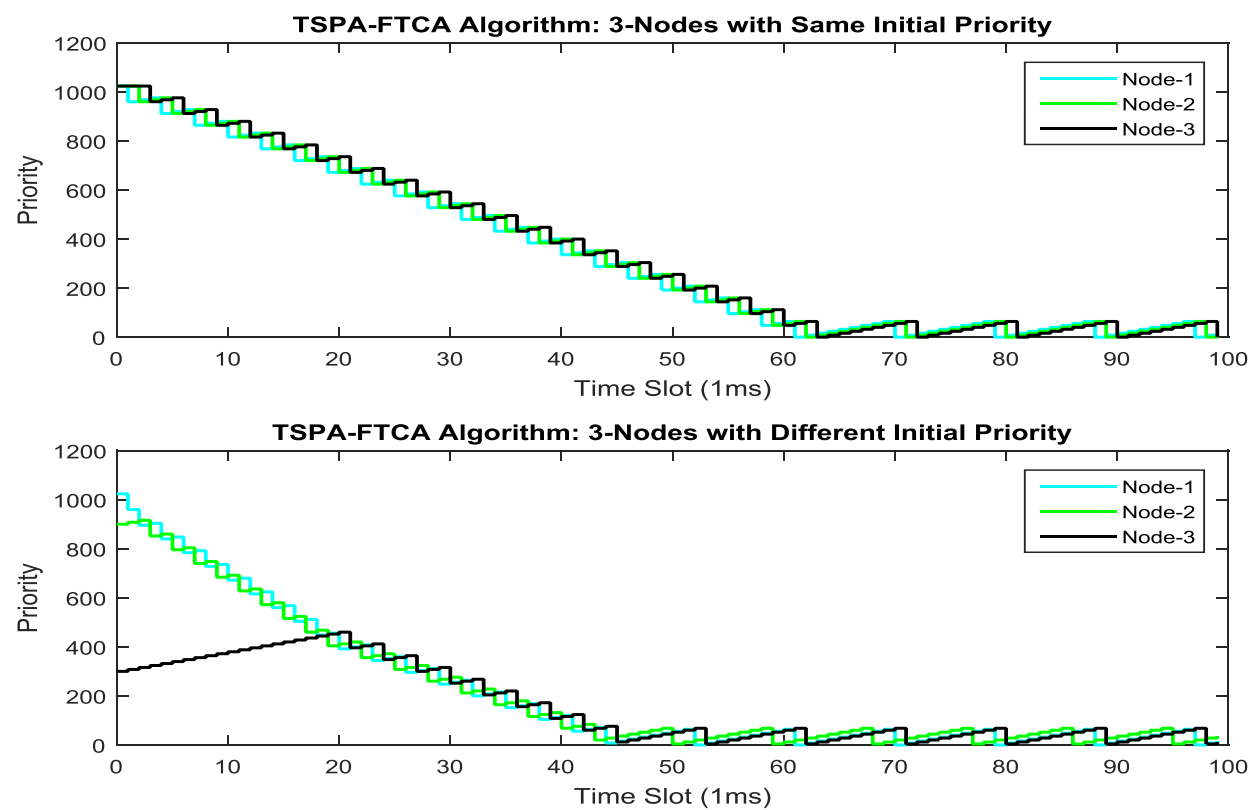

Figure 9: TSPA-FTCA Algorithm in a Three-Node Configuration. 


\subsection{Fault-Tolerance and Task Migration Operations Algorithm}

An initial algorithm supporting fault tolerance and task migration was introduced in $[68,69,71]$. That algorithm was implemented inside an AMFT block and provided functionality to detect, isolate and reconfigure the distributed system in case of a node's failure. The Fault-Tolerance and Task Migration Operations (FTTMO) algorithm, proposed in this section, builds upon the earlier version [68, 69, 71], offering the additional functions of node level fault-detection and self-registration. Moreover, it utilizes the TSPA-FTCA algorithm specified in section 6.1 .

The Fault-Tolerance and Task Migration Operations (FTTMO) algorithm, presented in this section, has four main phases: initialization, node registration, normal operation, and fault handling. The algorithm assumes that the TSPA-FTCA algorithm, defined in section 6.1, is in operation. In addition, it assumes that the tasks are grouped in sets, using an optimal scheduler, which takes into account the task dependencies and the corresponding communications overhead. All task sets are pre-allocated to the nodes and the test scenarios depend on the available number of nodes. The number of the pre-configured scenarios should be optimal. Table 3 shows the task-sets-to-nodes assignment scenarios in case of a three-node distributed system, where task set-1 is executed by node- 1 , task set- 2 by node- 2 and task set- 3 by node- 3 . The functions and logic flow of the FTTMO algorithm are represented by the pseudo code in Listing 2 .

TABLE 3: TASK-SETS-TO-NODES ASSIGNMENT SCENARIOS

\begin{tabular}{|c|l|c|c|c|}
\hline Scenario \# & \multicolumn{1}{|c|}{ Description } & Node-1 & Node-2 & Node-3 \\
\hline 1 & All three nodes are healthy & Task Set-1 & Task Set-2 & Task Set-3 \\
\hline 2 & $\begin{array}{l}\text { Node-3 fails, Node-1 and Node-2 are } \\
\text { healthy }\end{array}$ & $\begin{array}{l}\text { Task Set-1, } \\
\text { Task Set-3 }\end{array}$ & Task Set-2 & N/A \\
\hline 3 & $\begin{array}{l}\text { Node-1 fails, Node-2 and Node-3 are } \\
\text { healthy }\end{array}$ & N/A & $\begin{array}{l}\text { Task Set-2, } \\
\text { Task Set-1 }\end{array}$ & Task Set-3 \\
\hline 4 & $\begin{array}{l}\text { Node-2 fails, Node-1 and Node-3 are } \\
\text { healthy }\end{array}$ & Task Set-1 & N/A & $\begin{array}{l}\text { Task Set-2, } \\
\text { Task Set-3 }\end{array}$ \\
\hline
\end{tabular}

Listing 2: FAUlt-ToleranCe AND TASk MigRation Operations Algorithm (FTTMO)

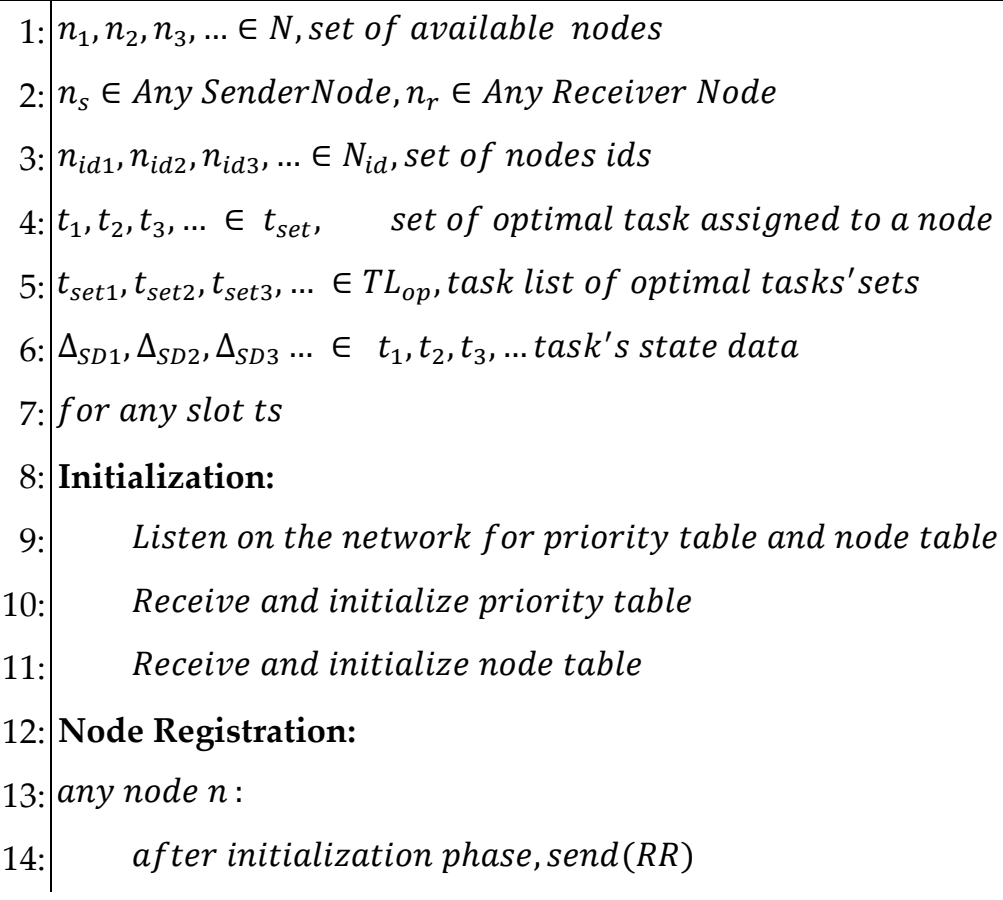




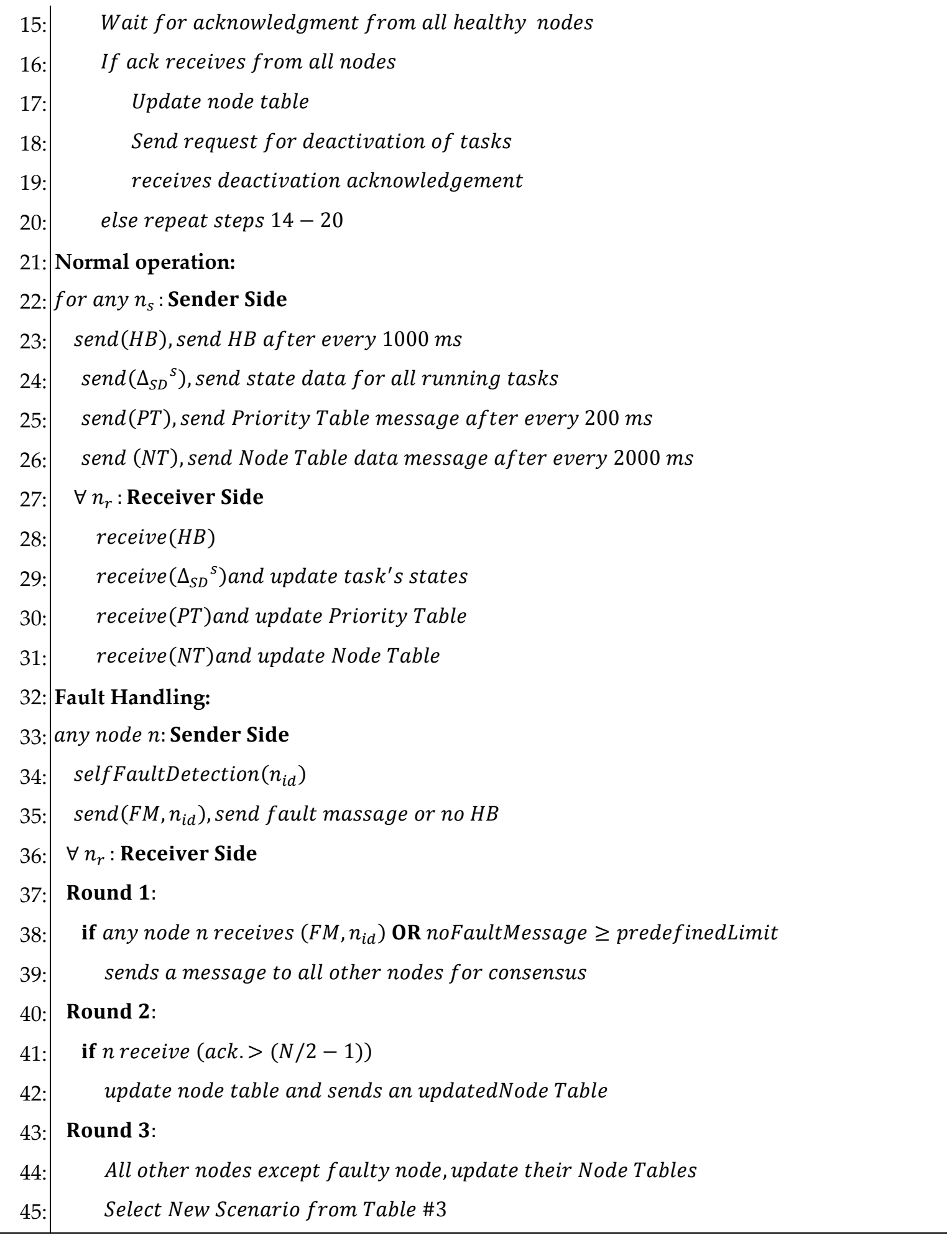

The details of each phase of the FTTMO algorithm are explained in the following sections.

\subsubsection{Initialization Phase}

The initialization phase of the algorithm (lines 8 -11, Listing 2) is shown in Figure 10. After the start-up, an entering node $n$ listens on the network for a set of messages, which includes the local view of priorities table, 
Heartbeat (HB) and node table messages. The local view of priorities tables message contains communication priority information for all nodes in the distributed system. It is essential for the entering node $n$ to know about the communication schedule before transmitting on the channel to avoid a possible collision. Based on this information, the entering node $n$ sets up its own local view priority table and sets its own priority value to the maximum value of 1024. In addition, the entering node also sets up its own node table by receiving a node table message from node-2 as shown in Figure 10. A node table message, as shown in Table 4, contains information regarding the total number of nodes present, the assigned task set to each node and their statuses. The total number of nodes in a node table represents the maximum number of nodes which can be registered. After the setup of the priority table and node table, the entering node can send a request for registration. The process of registration is described in section 6.2.2 below. If there is no message until up to three seconds (3000 time slots), the entering node starts sending its own set of messages (local view of priority table message, Heartbeat message and node table message).

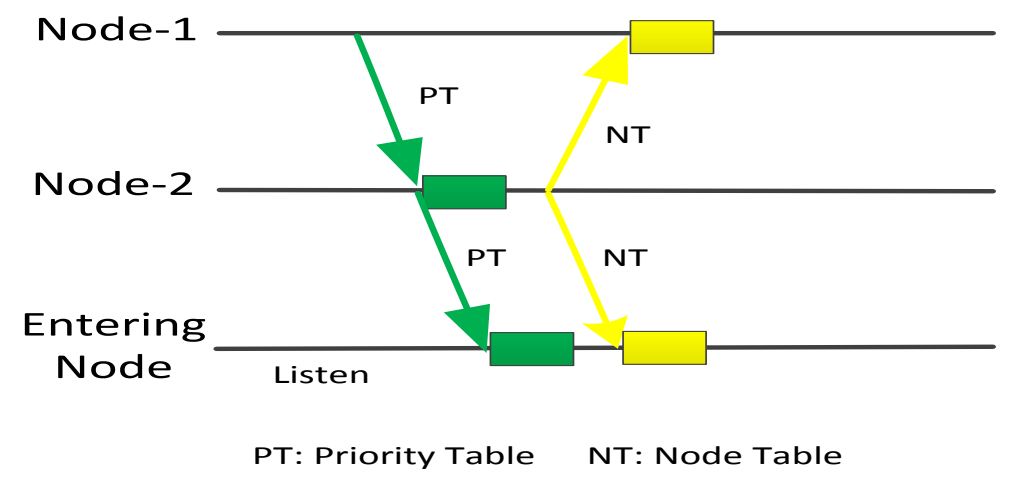

Figure 10: Initialization Phase.

TABLE 4: NODE TABLE OF NODE-3 AFTER INITIALIZATION

\begin{tabular}{|c|c|c|c|}
\hline \multicolumn{4}{|c|}{ Node Table } \\
\hline Total Nodes (N) & $\mathbf{3}$ & Total Scenarios & $\mathbf{4}$ \\
\hline \multicolumn{2}{|c|}{ Total Active Node } & $\mathbf{2}$ & \multicolumn{2}{c|}{ Scenario \# } & $\mathbf{2}$ \\
\hline Inactive Node \# & $\mathbf{3}$ & Transmission Node \# & Node Status \\
\hline Node-ID & \multicolumn{2}{|c|}{ Assigned Task Set } & Active and Healthy \\
\hline 1 & Task Set-1, Task Set-3 & Active and Healthy \\
\hline 2 & Task Set-2 & \\
\hline 3 & NIL &
\end{tabular}

\subsubsection{Node Registration Phase}

During this phase (lines $12-20$, Listing 2) a new node can be added to the distributed system. The process of adding a node is called a node registration. After the initialization phase, the entering node sends a request for registration (RR), as shown in Figure 11. All the other nodes (e.g. node-1, node-2) in the network receive its request and send an acknowledgement message to indicate that a new node is available in the network. Once all the acknowledgments are received, the entering node updates its node table as shown in Table 5. Following that, a task set is assigned to the new entering node. The process of the task assignment is done in two steps, if tasks are already assigned to another node, first these are deactivated. As shown in Table 5 , task set- 1 ' $t_{\text {set } 1}$ ' and task set-3 ' $t_{\text {set } 3}$ ' are assigned to node- 1 . Therefore, the entering node sends a deactivation request to the node- 1 , which is currently executing task set-3 ' $t_{\text {set } 3}$ '. On receipt of the deactivation message, node- 1 deactivates task set-3 ' $t_{\text {set } 3}$ ' and updates its node table by removing task set-3 ' $t_{\text {set } 3}$ '. As soon as node-3 receives an acknowledgement from node- 1 for the deactivation of task set- 3 ' $t_{\text {set }}$ ', the entering node (node-3) starts executing tasks as shown in Table 6. On the other hand, if task set-3 ' $t_{\text {set } 3}$ ' is not assigned to any node, the entering node (node-3) can pro- 
ceed to executing task set-3 ' $t_{\text {sets }}$ ' immediately.

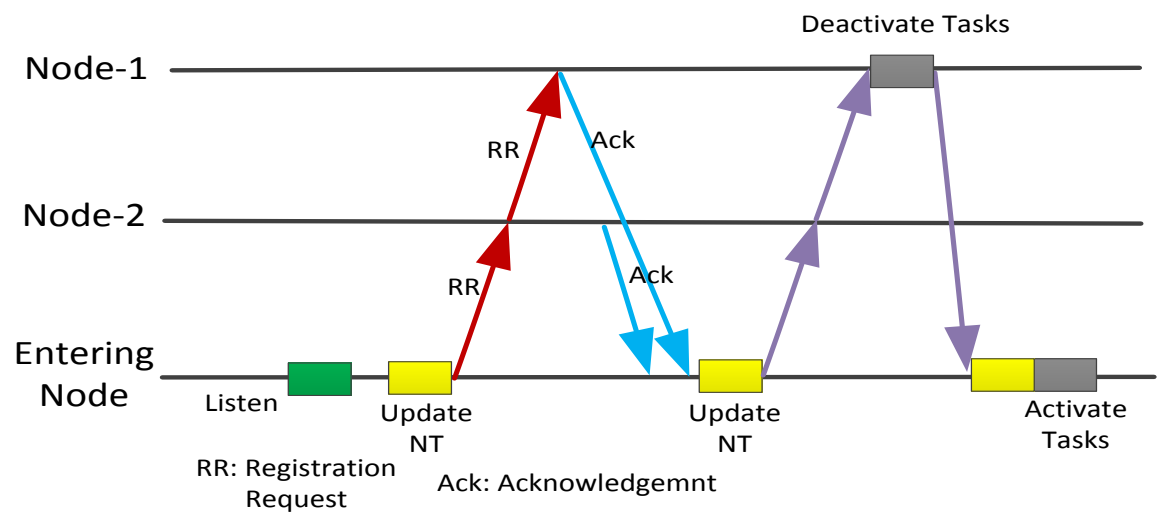

Figure 11: Node Registration and Task Activation/Deactivation.

TABLE 5: NODE TABLE OF NODE-3 AFTER All SUCCESSFul ACKNOWLEDGEMENTS

\begin{tabular}{|c|c|c|c|c|c|}
\hline \multicolumn{6}{|c|}{ Node Table } \\
\hline \multicolumn{2}{|c|}{ Total Nodes (N) } & 3 & & & 4 \\
\hline \multicolumn{2}{|c|}{ Total Active Node } & 3 & & & 1 \\
\hline \multicolumn{2}{|c|}{ Inactive Node \# } & - & Tra & & 1 \\
\hline Node-ID & \multicolumn{3}{|c|}{ Assigned Task Set } & & Node Status \\
\hline 1 & \multicolumn{3}{|c|}{ Task Set-1, Task Set-3 } & \multicolumn{2}{|c|}{ Active and Healthy } \\
\hline 2 & \multicolumn{3}{|c|}{ Task Set-2 } & \multicolumn{2}{|c|}{ Active and Healthy } \\
\hline 3 & \multicolumn{3}{|c|}{ NIL } & \multicolumn{2}{|c|}{ Active and Healthy } \\
\hline
\end{tabular}

TABle 6: Node TABLE OF NODE-3 AFTER TASK DeActivation CONFIRMATION FROM NODE-2

\begin{tabular}{|c|c|c|c|c|c|}
\hline \multicolumn{6}{|c|}{ Node Table } \\
\hline \multicolumn{2}{|c|}{ Total Nodes (N) } & 3 & & Total Scena & 4 \\
\hline \multicolumn{2}{|c|}{ Total Active Node } & 3 & & Scenario \# & 1 \\
\hline \multicolumn{2}{|c|}{ Inactive Node \# } & - & & ismission $\mathbf{N}$ & 1 \\
\hline Node-ID & \multicolumn{3}{|c|}{ Assigned Task Set } & & Node Status \\
\hline 1 & \multicolumn{3}{|c|}{ Task Set-1 } & & Active and Healthy \\
\hline 2 & \multicolumn{3}{|c|}{ Task Set-2 } & & Active and Healthy \\
\hline 3 & \multicolumn{3}{|c|}{ Task Set-3 } & & Active and Healthy \\
\hline
\end{tabular}

\subsubsection{Normal Operation Phase}

During the normal operation (lines $21-31$, Listing 2) each node is bound to send a Heartbeat (HB) message, which indicates the health status of the node, as shown in Figure 12. On receipt of the HB message, the receiving node assumes that the node is active and executes its task's set ' $t_{\text {set }}$ '. For the purpose of simulating the FTTMO algorithm, a suitable timing scheme is used. The value of the sending period $T$ of the HB message is carefully chosen, as it indirectly affects the system reconfiguration time in case of a failure. A short time interval for the sending period $T$ is preferred, however, it leads to an increased demand for access to the wireless communication channel. Therefore, a time of $500 \mathrm{~ms}$ is selected. Each node sends a HB message within $500 \mathrm{~ms}$. If a node $n$ is unable to send the HB message within $500 \mathrm{~ms}$, it is suspected as failed. In addition to the HB message, every node also exchanges their priority table and node table messages. The priority table message is sent every 200 
$\mathrm{ms}$ while the node table message is sent every $2000 \mathrm{~ms}$. The choice of the small periodic time of $200 \mathrm{~ms}$ to synchronize the local view of the priority table helps to avoid a collision in the network. The node table message, which is exchanged every $2000 \mathrm{~ms}$, keeps all the node tables synchronized. To retain the task state in a case of a failure, the task state data is checkpointed and transmitted to the other nodes for storage on a regular basis. To minimize the amount of the task state data, only the output results of a task is stored. The sending period for the task state depends on the application task period.

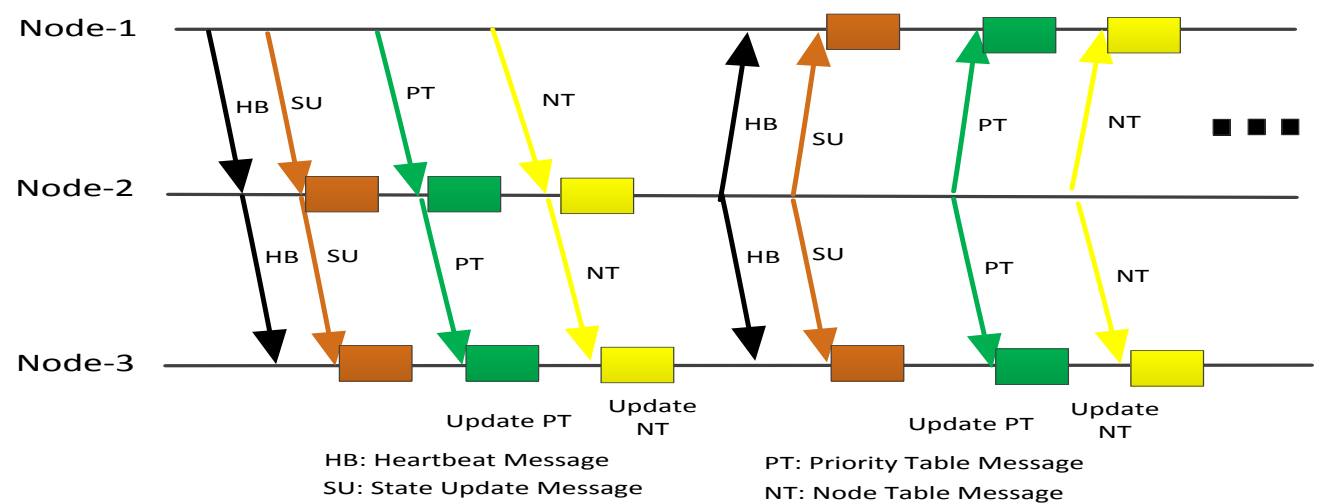

Figure 12: Normal Operation.

\subsubsection{Fault Handling Phase}

During the fault handling phase (lines $32-45$, Listing 2) the failure of a node is detected by the node level faultdetection mechanism. A faulty node $k$ has either a fail-silent behaviour or it can send a fault message to other nodes. If a node $n$ receives neither a fault message nor a HB message from a node $k$, it "suspects" that the node $k$ has failed. However, a node $n$ only confirms a node $k$ as a failed node, if it does not receive a fault message or a HB message from a node $k$ for at least five times consecutively. It that case, it sends a message to all other nodes informing them about the failure of the node $k$. If the other nodes form the same "opinion" about a node $k$ (based on receiving neither fault messages nor HB messages from it for at least three times consecutively), then all nodes send acknowledgments to the node $n$. On receipt of at least (N/2-1) such acknowledgements, the node $n$ updates its node table correspondingly and sends an updated node table message to all other $N$-1 nodes. Following that, all healthy nodes update their node tables and select a task set according to the available nodes.

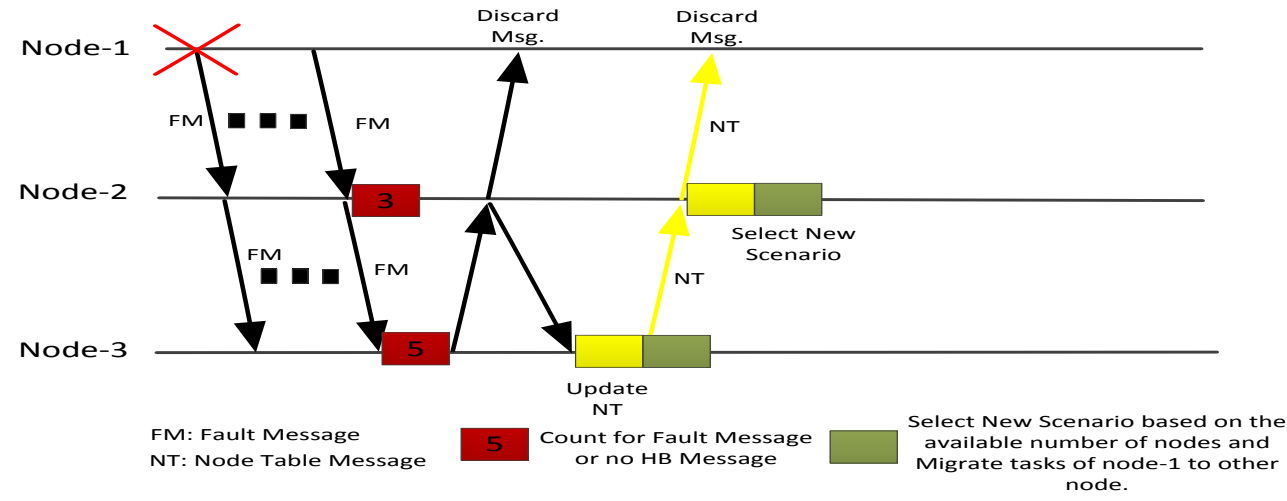

Figure 13: Fault Handling and Task Migration.

Let us consider the three-node fault-handling scenario, illustrated in Figure 13, which assumes that node-1 is faulty and capable of sending a periodic fault message (FM) instead of a HB message to the other nodes. The 
length of the period of sending the FM is similar to that of the HB message (500 ms). The node- 3 and node- 2 suspect the failure of node- 1 . However, due to a communication error, node- 2 is unable to receive all the FM messages and misses two of the five messages. As soon as the count of the node- 3 FM messages reaches five, it sends a message to the other nodes that node- 1 has failed. Based on this message, node- 2 sends an acknowledgement message to node- 3 . This acknowledgement signifies that node- 2 also has the same "opinion" about node- 1 . Afterwards, node- 3 marks node- 1 as a failed node in the node table and sends an updated node table message. On receipt of the updated node table, both nodes (node-2 and node-3) select scenario \#3 from Table 3.

\section{CONCLUSIONS}

Current redundancy based spaceborne distributed computing systems, where computing tasks are bound to execute on a primary or its peer redundant node, cannot provide a computationally efficient, reconfigurable and highly reliable computing platform. The computational efficiency is limited to the computing power of a single node and the fault-tolerance is provided by a single peer redundant node. These redundant resources cannot be utilized by any other computing subsystems, making the distributed system highly inefficient in terms of resource utilization.

A novel approach to distributed computing called next-generation spacecraft fault-tolerant distributed computing (NGS-FTDC) platform is proposed. The NGS-FTDC approach is resource efficient where tasks are migrated and computing nodes are reconfigured to protect against a node failure. This makes the distributed system more reliable and computationally efficient. Instead of a wired interconnection network, a wireless network is proposed for the communication among the distributed nodes. Traditional wireless protocols do not provide deterministic and fault-tolerant behavior. Therefore a novel a Time-Slot Priority Adaptive Fault-Tolerant Channel Access (TSPA-FTCA) algorithm is proposed, which uses an adaptive prioritizing channel access policy for deterministic access to the channel, with each message being triplicated to protect against packet loss.

To provide an architectural level fault-tolerance for the NGS-FTDC platform, a novel Fault-Tolerance and Task Migration Operations (FTTMO) algorithm is also proposed. The FTTMO algorithm detects a failure of a computing node and migrates the tasks to other nodes in a pure distributed setting. In addition, this algorithm allows the NGS-FTDC platform to add additional computing nodes on the fly to restore / augment the computational capacity and system reliability.

In conclusion, this paper highlighted the demands of future applications and the importance of the development of a new distributed computing platform aimed at future space missions, particularly designed with wireless interconnection links. The proposed distributed computing platform meets the desired requirements, thus setting a benchmark for further improvements.

\section{ACKNOWLEDGEMENTS}

Funding from the University of Leicester, UK, and the FP7 Space grant "ReVuS: Reducing the vulnerability of space systems" is gratefully acknowledged.

\section{REFERENCES}

[1] A. Helmerich, et al., "Study of Worldwide Trends and R\&D Programmes in Embedded Systems.," Fast GmbH, Munich Germany, 2005.

[2] A. Sehmi, "On distributed embedded systems," J. ACSIJ, vol. 2, Issue 1, No. 2, Jan. 2013.

[3] H. Kopetz, et al., "Distributed fault-tolerant real-time systems: the Mars approach," IEEE Micro, vol. 9 , pp. 25-40, 1989.

[4] A. S. Tanenbaum and M. v. Steen, "Fault Tolerance," in Distributed systems : principles and paradigms, 2nd ed Upper Saddle RIiver, N.J.: Pearson Prentice Hall, 2007.

[5] "Distributed systems," in A Review of Ada Tasking. vol. 262, A. Burns, et al., Eds., ed: Springer Berlin Heidelberg, 1987, pp. 63-72.

[6] C. Georgiou and A. A. Shvartsman, Do-All Computing in Distributed Systems: Cooperation in the Presence of Adversity: Springer, 2008.

[7] C. Georgiou and A. A. Shvartsman, Cooperative Task-Oriented Computing Algorithm and Complexity, 
1st ed.: Morgan \& Claypool, 2011.

[8] S. Buckreuss and M. Zink, "The missions TerraSAR-X and TanDEM-X: Status, challenges, future perspectives," in General Assembly and Scientific Symp., XXXth URSI, 2011, pp. 1-1.

[9] M. Zink and A. Moreira, "TanDEM-X mission: Overview, challenges and status," in Proc. International Geoscience and Remote Sensing Symp., 2013, pp. 1885-1888.

[10] J. Kichun, et al., "Development of Autonomous Car-Part I: Distributed System Architecture and Development Process," IEEE Trans. Ind. Electron., vol. 61, pp. 7131-7140, 2014.

[11] C. Villalpando, et al., "Reliable multicore processors for NASA space missions," in Proc. Aerospace Conf., Big Sky, MT, USA, 2011, pp. 1-12.

[12] D. A. Rennels, "Architectures for fault-tolerant spacecraft computers," Proc. IEEE, vol. 66, pp. 1255$1268,1978$.

[13] G. Klančar, et al., "Image-Based Attitude Control of a Remote Sensing Satellite," J. Intell Robot Syst, vol. 66, pp. 343-357, May 2012.

[14] J. D. Ruiz. (2013). Overcoming the Embedded CPU Performance Wall. Available:

http://www.embedded.com/design/mcus-processors-and-socs/4405280/Overcoming-the-embedded-CPUperformance-wall-

[15] Y. Xie and B. Zhu, "Architecture design of spaceborne SAR imaging processing system," in Proc. Int. Conf. of Signal Processing, 2010, pp. 2283-2286.

[16] Gutierrez-Nava, et al., "TOPMEX-9 DISTRIBUTED SAR MISSION EMPLOYING

NANOSATELLITE CLUSTER," presented at the 63rd Int. Astronautical Congr., Naples, Italy., 2012.

[17] P. Thambidurai and P. You-keun, "Interactive consistency with multiple failure modes," in Proc. Reliable Distributed Systems, Seventh Symp., Columbus, OH, 1988, pp. 93-100.

[18] R. W. Butler, "A primer on architectural level fault tolerance " Langley Research Center, Hampton, Virginia, 2008.

[19] J. Arlat, Crouzet, Y., Deswarte, Y., Laprie, J.-C., Powell, D., David, P., Dega, J. L., Rabéjac, C., Schindler, H. and Soucailles,J.-F, FAULT TOLERANT COMPUTING: Wiley Encyclopedia of Electrical and Electronics Engineering, 1999.

[20] A. Aviziens, "Fault-Tolerant Systems," IEEE Trans. Comput., vol. 25, pp. 1304-1312, 1976.

[21] H. Kopetz, Real-Time Systems: Design Principles for Distributed Embedded Applications, 1998 ed.: Kluwer Academic Publishers. , 1997.

[22] S. D'Angelo, et al., "Transient and permanent fault diagnosis for FPGA-based TMR systems," in Proc. Int. Defect and Fault Tolerance in VLSI Systems Symp., 1999, pp. 330-338.

[23] J. F. Wakerly, "Transient Failures in Triple Modular Redundancy Systems with Sequential Modules," IEEE Trans. Comput., vol. C-24, pp. 570-573, 1975.

[24] W. Xinsheng and S. Hanxu, "Fault tolerance design on onboard computer using COTS components," in Proc. Systems and Control in Aerospace and Astronautics, 1st Int. Symp., 2006, pp. 3 pp.-1224.

[25] R. E. Lyons and W. Vanderkulk, "The Use of Triple-Modular Redundancy to Improve Computer Reliability," J. IBM Research and Development, vol. 6, pp. 200-209, 1962.

[26] J. R. Sklaroff, "Redundancy Management Technique for Space Shuttle Computers," J. IBM Research and Development, vol. 20, pp. 20-28, 1976.

[27] H. Pham, "Optimal design of hybrid fault-tolerant computer systems," J. Mathematical and Computer Modelling, vol. 16, pp. 29-33, 1992.

[28] P. K. Samudrala, et al., "Selective triple Modular redundancy (STMR) based single-event upset (SEU) tolerant synthesis for FPGAs," IEEE Trans. Nucl. Sci., vol. 51, pp. 2957-2969, 2004.

[29] B. Pratt, et al., "Improving FPGA Design Robustness with Partial TMR," in Proc. Reliability Physics, 44th Аnпu. Symp., 2006, pp. 226-232. 
[30] F. L. Kastensmidt, et al., "On the optimal design of triple modular redundancy logic for SRAM-based FPGAs," in Proc. Design, Automation and Test in Europe, 2005, pp. 1290-1295 Vol. 2.

[31] F. Lima, et al., "Designing fault tolerant systems into SRAM-based FPGAs," in Proc. Design Automation Conf., 2003, pp. 650-655.

[32] R. Guerraoui and A. Schiper, "Fault-Tolerance by replication in Distributed systems " in Proc. Reliable Software Technologies, Ada-Europe, 1996, pp. 38-57.

[33] A. Girault, et al., "An Active Replication Scheme That Tolerates Failures in Distributed Embedded Real-Time Systems," in Design Methods and Applications for Distributed Embedded Systems. vol. 150, B. Kleinjohann, et al., Eds., ed: Springer US, 2004, pp. 83-92.

[34] M. Pease, et al., "Reaching Agreement in the Presence of Faults," J. ACM, vol. 27, pp. 228-234, 1980.

[35] L. Lamport, et al., "The Byzantine Generals Problem," ACM Trans. Program. Lang. Syst., vol. 4, pp. 382-401, 1982.

[36] U. Schmid, et al., "Formally verified Byzantine agreement in presence of link faults," in Proc. Distributed Computing Systems conf., 2002, pp. 608-616.

[37] S. Hin-Sing, et al., "Byzantine agreement in the presence of mixed faults on processors and links," IEEE Trans. Parallel and Distrib. Syste., vol. 9, pp. 335-345, 1998.

[38] F. B. Schneider and Z. Lidong, "Implementing trustworthy services using replicated state machines," IEEE J. Security \& Privacy vol. 3, pp. 34-43, 2005.

[39] M. Chereque, et al., "Active replication in Delta-4," in Proc. Fault-Tolerant Computing Twenty-Second Int. Symp., 1992, pp. 28-37.

[40] C. Marchetti, et al., "Fully distributed three-tier active software replication," IEEE Trans. Parallel and Distrib. Syste. , vol. 17, pp. 633-645, 2006.

[41] R. Baldoni, et al., "Asynchronous active replication in three-tier distributed systems," in Proc. Dependable Computing, Pacific Rim Int. Symp., 2002, pp. 19-26.

[42] R. Baldoni, et al., "Active software replication through a three-tier approach," in Proc. Reliable Distributed Systems, 21st IEEE Symp., 2002, pp. 109-118.

[43] D. Powell, "Distributed Fault-Tolerance," in Delta-4: A Generic Architecture for Dependable Distributed Computing. vol. 1, D. Powell, Ed., ed: Springer Berlin Heidelberg, 1991, pp. 89-124.

[44] N. Budhiraja and K. Marzullo, "Tradeoffs in implementing primary-backup protocols," in Proc. Parallel and Distributed Processing, Seventh IEEE Symp., 1995, pp. 280-288.

[45] Z. Hengming and F. Jahanian, "A real-time primary-backup replication service," IEEE Trans. Parallel and Distrib. Syste., vol. 10, pp. 533-548, 1999.

[46] A. M. Deplanche, et al., "Implementing a semi-active replication strategy in CHORUS/ClassiX, a distributed real-time executive," in Proc. Reliable Distributed Systems, 18th IEEE Symp., 1999, pp. 90-101.

[47] X. Defago, et al., "Semi-passive replication," in Proc. Reliable Distributed Systems, Seventeenth IEEE Symp., 1998, pp. 43-50.

[48] X. Défago and A. Schiper, "Semi-passive replication and Lazy Consensus," J. Parallel Distrib. Comp., vol. 64, pp. 1380-1398, 2004.

[49] X. Défago and A. Schiper, "Specification of replication techniques, semi-passive replication, and lazy consensus. ," Japan Advanced Institute of Science and Technology, Ishikawa, Japan KS-RR-2002-001, 2002.

[50] T. D. Chandra and S. Toueg, "Unreliable failure detectors for reliable distributed systems," J. ACM, vol. 43, pp. 225-267, 1996.

[51] M. Hecht, et al., "A distributed fault tolerant architecture for nuclear reactor and other critical process control applications," in Proc. Twenty-First Int. Fault-Tolerant Computing Symp., 1991, pp. 462-498.

[52] D. Nguyen and L. Dar-Biau, "Recovery blocks in real-time distributed systems," in Proc. Reliability and Maintainability Annu. Symp. , 1998, pp. 149-154. 
[53] V. Izosimov, et al., "Design optimization of time- and cost-constrained fault-tolerant distributed embedded systems," in Proc. Design, Automation and Test in Europe, 2005, pp. 864-869 Vol. 2.

[54] M. N. Lovellette, et al., "Strategies for fault-tolerant, space-based computing: Lessons learned from the ARGOS testbed," in Proc. Aerospace IEEE Conf. , 2002, pp. 5-2109-5-2119 vol.5.

[55] P. Subramanyan, et al., "Multiplexed redundant execution: A technique for efficient fault tolerance in chip multiprocessors," in Proc. Design, Automation \& Test in Europe Conf., 2010, pp. 1572-1577.

[56] H. Kopetz, et al., "Fault-Tolerant Membership Service in a Synchronous Distributed Real-Time System," in Dependable Computing for Critical Applications. vol. 4, A. Avižienis and J.-C. Laprie, Eds., ed: Springer Vienna, 1991, pp. 411-429.

[57] K. H. Kim and E. Shokri, "Minimal-delay decentralized maintenance of processor-group membership in TDMA-bus LAN systems," in Proc. Distributed Computing Systems, 13th Int. Conf., 1993, pp. 410-419.

[58] K. H. Kim and C. Subbaraman, "Dynamic configuration management in reliable distributed real-time information systems," IEEE Trans. Knowl. Data Eng., vol. 11, pp. 239-254, 1999.

[59] X. Olive, "FDI(R) for satellites: How to deal with high availability and robustness in the space domain?," Int. J. Appl. Math. Comput. Sci., vol. 22, pp. 99-107, 2012.

[60] W. Harkin, "Utilize FDIR Design Techniques to provide for Safe and Maintainable On-Orbit Systems," Johnson Space Center, Technique DFE-7, 1994.

[61] O. Emam, et al., "A fault detection, isolation and recovery (FDIR) strategy based on a message exchange approach to implement autonomous FDIR management on the MARC system.," in Proc. Data Systems In Aerospace, Budapest, 2010, p. 33.

[62] B. Jackson, "A robust fault protection architecture for low-cost nanosatellites," in Proc. IEEE Aerospace Conf., 2014, pp. 1-8.

[63] J. H. Lala, et al., "Advanced Information Processing System (AIPS)-based fault tolerant avionics architecture for launch vehicles," in Proc. IEEE/AIAA/NASA 9th Digital Avionics Systems Conf., 1990, pp. 125132.

[64] D. A. Rennels, "Reconfigurable Modular Computer Networks for Spacecraft On-Board Processing," IEEE Computer, vol. 11, pp. 49-59, 1978.

[65] S. N. Chau, et al., "Design of a fault-tolerant COTS-based bus architecture," IEEE Trans. Reliab., vol. 48, pp. 351-359, 1999.

[66] D. A. Rennels, et al., "A fault-tolerant embedded microcontroller testbed," in Proc. Fault-Tolerant Systems Pacific Rim Int. Symp., 1997, pp. 7-14.

[67] D. Powell, "Distributed fault tolerance: lessons from Delta-4," IEEE Micro, vol. 14, pp. 36-47, 1994.

[68] M. Fayyaz and T. Vladimirova, "Fault-Tolerant Distributed approach to satellite On-Board Computer design," in Proc. IEEE Aerospace Conf., 2014, pp. 1-12.

[69] M. Fayyaz, et al., "Adaptive middleware design for satellite fault-tolerant distributed computing," in Proc. IEEE NASA/ESA Adaptive Hardware and Systems (AHS) Conf., 2012, pp. 23-30.

[70] T. Vladimirova and M. Fayyaz, "Fault-Tolerant Computing on Board Spacecraft using Distributed Multicore Processors," submitted to Acta Astronautica,Elsevier, 2015.

[71] T. Vladimirova and M. Fayyaz, "Wireless Fault-Tolerant Distributed Architecture for Satellite Platform Computing," in Convergence and Hybrid Information Technology, ed: Springer, 2012, pp. 428-436.

[72] Lu, et al., "OBC-NG: Towards a reconfigurable on-board computing architecture for spacecraft," in Aerospace Conference, 2014 IEEE, 2014, pp. 1-13.

[73] M. M. Ibrahim, et al., "Reconfigurable fault tolerant avionics system," in Proc. IEEE Aerospace Conf., 2013, pp. 1-12.

[74] A. Jacobs, et al., "Reconfigurable Fault Tolerance: A Comprehensive Framework for Reliable and Adaptive FPGA-Based Space Computing," ACM Trans. Reconfigurable Technol. Syst., vol. 5, pp. 1-30, 2012. 
[75] K. P. Gostelow, "The design of a fault-tolerant, real-time, multi-core computer system," in Proc. IEEE Aerospace Conf., 2011, pp. 1-8.

[76] A. Shye, et al., "PLR: A Software Approach to Transient Fault Tolerance for Multicore Architectures," IEEE Trans. Dependable Secure Comput., vol. 6, pp. 135-148, 2009.

[77] N. Aggarwal, et al., "Configurable isolation: building high availability systems with commodity multicore processors," SIGARCH Comput. Archit. News, vol. 35, pp. 470-481, 2007.

[78] C. LaFrieda, et al., "Utilizing Dynamically Coupled Cores to Form a Resilient Chip Multiprocessor," in Proc. IEEE/IFIP Int. Dependable Systems and Networks Conf., 2007, pp. 317-326.

[79] S. S. Mukherjee, et al., "Detailed design and evaluation of redundant multi-threading alternatives," in Proc. 29th Annu Int. Computer Architecture Symp., 2002, pp. 99-110.

[80] J. Ramos, et al., "High-performance, Dependable Multiprocessor," in Proc. IEEE Aerospace Conf., 2006, p. 13 pp.

[81] J. Samson, et al., "High Performance Dependable Multiprocessor II," in IEEE Aerospace Conf., 2007, pp. $1-22$.

[82] J. Samson, et al., "Technology Validation: NMP ST8 Dependable Multiprocessor Project II," in IEEE Aerospace Conf., 2007, pp. 1-18.

[83] J. R. Samson, Jr., "Implementation of a Dependable Multiprocessor CubeSat," in IEEE Aerospace Conf., 2011, pp. 1-10.

[84] W. Gropp, et al., Beowulf Cluster Computing with Linux, 2nd ed.: MIT Press, 2003.

[85] I. V. McLoughlin and T. R. Bretschneider, "Reliability through redundant parallelism for micro-satellite computing," ACM Trans. Embed. Comput. Syst., vol. 9, pp. 1-25, 2010.

[86] D. S. Katz and P. L. Springer, "Development of a spaceborne embedded cluster," in Proc. IEEE Int. Cluster Computing Conf., 2000, pp. 119-123.

[87] D. Ngo and M. Harris, "A reliable infrastructure based on COTS technology for affordable space application," in IEEE Aerospace Conf., 2001, pp. 2435-2441 vol.5.

[88] C. Steiger, et al., "GOCE end-of-mission operations report," ESA2014.

[89] L. D. Friedman, "Phobos-Grunt Failure Report Released," vol. 2015, ed, 2012.

[90] J. Eickhoff, Onboard Computers, Onboard Software and Satellite Operations, An Introduction: Springer-Verlag Berlin Heidelberg 2012.

[91] F. Kraja, et al., "Designing Spacecraft High Performance Computing Architectures," in Advanced Computing. vol. 93, M. Bader, et al., Eds., ed: Springer Berlin Heidelberg, 2013, pp. 137-156.

[92] D. S. Katz and R. R. Some, "NASA advances robotic space exploration," Computer, vol. 36, pp. 52-61, 2003.

[93] T. Sterling, et al., "High Performance Computing Systems for Autonomous Spaceborne Missions," Int. J. High Perform. Comput. Appl., vol. 15, pp. 282-296, 2001.

[94] S. Fuchs and A. J. Wardrop, "Fault Tolerant Computer System," USA Patent, 2000.

[95] J. K. Kishore, et al., "A real time fault tolerant microprocessor based On-Board Computer System for INSAT-2 spacecraft," in Formal Techniques in Real-Time and Fault-Tolerant Systems. vol. 863, H. Langmaack, et al., Eds., ed: Springer Berlin Heidelberg, 1994, pp. 476-487.

[96] W. H. Zheng and J. T. Armstrong, "Wireless intra-spacecraft communication: The benefits and the challenges," in Adaptive Hardware and Systems (AHS), 2010 NASA/ESA Conference on, 2010, pp. 75-78.

[97] R. Aminiy, et al., "The Challenges of Intra-spacecraft Wireless Data Interfacing," in 58th International Astronautical Congress, 2007.

[98] Fink and P. W., "Wireless Network Communications Overview for Space Mission Operations," NASA CCSDS 880.0-G-1, December 2010. 
[99] K. Kimoon, et al., "Resource-conscious customization of CORBA for CAN-based distributed embedded systems," in Object-Oriented Real-Time Distributed Computing, 2000. (ISORC 2000) Proceedings. Third IEEE International Symposium on, 2000, pp. 34-41.

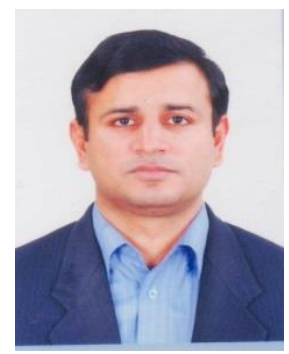

Muhammad Fayyaz received the BS degree in Electrical Engineering from University of Engineering and Technology (UET) Lahore, Pakistan, in 2004, the MSc in Electronics Engineering from the University of Surrey, Guildford, UK. He worked for 7 years in the Space Industry with hands-on experience on satellite subsystems to fly on board spacecraft. He is specialized in $O B C / O B D H, C C S D S$ based Telemetry and Telecommand subsystems and remote terminal units. He has successfully completed many national and international projects. He was the recipient of many awards including the doctoral fellowship from University of Leicester MS Scholarship holder from SUPARCO, and the best performer appreciation award for 2006-2011 from SUPARCO. At present, he is doing research on faulttolerant distributed computing Systems for space applications at the Department of Engineering, University of Leicester.

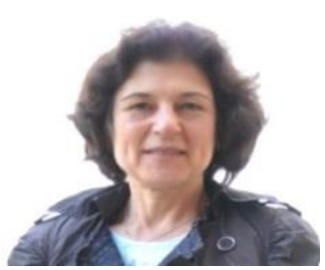

Tanya Vladimirova MEng, MSc, PhD, CEng, FIET, SMEEE, MACM, obtained the M.Sc. in Applied Mathematics from the Technical University of Sofia, Bulgaria, the MEng in Computer Systems Engineering, and the Ph.D. in VLSI Design from the St. Petersburg Electro-Technical University (LETI), Russia. She is currently Professor at the Department of Engineering, University of Leicester, UK. Prior to that, she was with the Surrey Space Centre, Department of Electrical and Electronic Engineering of the University of Surrey in Guildford, UK, where she led the VLSI Design and Embedded Systems research group. Her research interests are in the areas of intelligent image and data processing, distributed embedded computing, low-power VLSI design and wireless sensor networks. She acted as a general chair of the $9^{\text {th }}$ NASA/ESA Conference on Adaptive Hardware and Systems, AHS-2014 and a co-chair of the NASA conference on Military and Aerospace Applications of Programmable Logic Devices, MAPLD, from 2000 to 2006. 